\title{
On the Collision and Preimage Security of MDC-4 in the Ideal Cipher Model
}

\author{
Bart Mennink \\ Dept. Electrical Engineering, ESAT/COSIC and IBBT \\ Katholieke Universiteit Leuven, Belgium \\ bart.mennink@esat.kuleuven.be
}

\begin{abstract}
We present a collision and preimage security analysis of MDC-4, a 24 years old construction for transforming an $n$-bit block cipher into a $2 n$-bit hash function. We start with MDC-4 based on one single block cipher, and prove that any adversary with query access to the underlying block cipher requires at least $2^{5 n / 8}$ queries (asymptotically) to find a collision. For the preimage resistance, we present a surprising negative result: for a target image with the same left and right half, a preimage for the full MDC-4 hash function can be found in $2^{n}$ queries. Yet, restricted to target images with different left and right halves, we prove that at least $2^{5 n / 4}$ queries (asymptotically) are required to find a preimage. Next, we consider MDC-4 based on two independent block ciphers, a model that is less general but closer to the original design, and prove that the collision bound of $2^{5 n / 8}$ queries and the preimage bound of $2^{5 n / 4}$ queries apply to the MDC- 4 compression function and hash function design. With these results, we are the first to formally confirm that MDC-4 offers a higher level of provable security compared to MDC-2.
\end{abstract}

Keywords. MDC-4; double block length; hash function; collision resistance; preimage resistance.

\section{Introduction}

The focus of this work is the classical block cipher based hash function MDC-4. MDC-4 and its related hash function MDC-2 have first been described by Meyer and Schilling in 1988 [25], and have been patented by Brachtl et al. in 1990 [4]. MDC-2 has been standardized in ISO/IEC 10118-2 [13] and is used in numerous applications (see [15, 28] for an exposition), while MDC-4 is part of the IBM CLiC cryptographic module [6, 7]. In their original specification, MDC-2 and MDC-4 are instantiated using the block cipher DES. In this work, we step away from this design criterion and consider the designs based on any block cipher $E: \mathbb{Z}_{2}^{n} \times \mathbb{Z}_{2}^{n} \rightarrow \mathbb{Z}_{2}^{n}$ with key and message length $n$ bits (throughout, the first input to the block cipher is the key input).

MDC-2 is a Merkle-Damgård (MD) hash function design [5, 24] using a compression function $f_{\mathrm{MDC}-2}$ : $\mathbb{Z}_{2}^{3 n} \rightarrow \mathbb{Z}_{2}^{2 n}$ that internally calls the block cipher $E$ twice. It additionally employs two mappings $v$ and $w$, applied on the key inputs to the two block cipher calls. As $v$ and $w$ are originally constructed so as to have a distinct rang $\oint^{1}$, we can consider MDC-2 to be based on two block ciphers $E_{1}(\cdot, \cdot)=E(v(\cdot), \cdot)$ and $E_{2}(\cdot, \cdot)=E(w(\cdot), \cdot)\left[28\right.$. The compression function $f_{\mathrm{MDC}-2}: \mathbb{Z}_{2}^{3 n} \rightarrow \mathbb{Z}_{2}^{2 n}$ is defined as follows.

$$
\begin{aligned}
& f_{\mathrm{MDC}-2}(A, B, C) \\
& W \leftarrow E_{1}(A, C) \oplus C, \quad X \leftarrow E_{2}(B, C) \oplus C, \\
& Y \leftarrow W^{l}\left\|X^{r}, \quad Z \leftarrow X^{l}\right\| W^{r}, \\
& \text { return }(Y, Z) \text {. }
\end{aligned}
$$

Here, for a bit string $X$ of even length we denote by $X^{l}$ and $X^{r}$ its left and right halves. $f_{\mathrm{MDC}-2}$ can be considered as a parallel evaluation of two Matyas-Meyer-Oseas (MMO) constructions [22] followed by a swapping of the right halves of both states. Consequently, $f_{\mathrm{MDC}-2}$ does not achieve the desired level of security: finding a collision or a preimage for $f_{\mathrm{MDC}-2}$ is as hard as finding it for the two MMO constructions independently, hence requires about $2^{n / 2}$ or $2^{n}$ block cipher calls, respectively. For the full MDC-2 hash function, Knudsen et al. [15] demonstrated that roughly $2^{n} / n$ block cipher calls suffice for finding a collision, and about $2^{n}$ calls for finding a preimage. In 2007, Steinberger derived a nontrivial security lower bound on MDC-2 [28]. Steinberger considers the MDC-2 hash function using one single block cipher $E$ modeled as an ideal cipher, and proves that any adversary with query access to

\footnotetext{
1 The original specification (using block cipher DES) defines $v$ and $w$ as mappings from the ciphertext space to the key space, where the parity bits are omitted, and the second and third bits are set to 10 and 01 , respectively.
} 


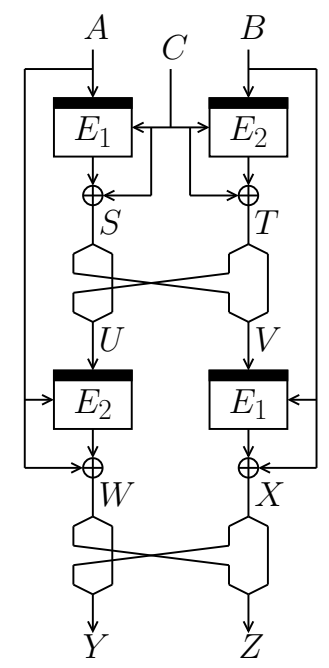

$$
\begin{aligned}
& f_{\mathrm{MDC}-4}(A, B, C) \\
& S \leftarrow E_{1}(A, C) \oplus C, \\
& T \leftarrow E_{2}(B, C) \oplus C, \\
& U \leftarrow T^{l} \| S^{r}, \\
& V \leftarrow S^{l} \| T^{r}, \\
& W \leftarrow E_{2}(U, A) \oplus A, \\
& X \leftarrow E_{1}(V, B) \oplus B, \\
& Y \leftarrow X^{l} \| W^{r}, \\
& Z \leftarrow W^{l} \| X^{r}, \\
& \operatorname{return}(Y, Z) .
\end{aligned}
$$

Fig. 1. The $f_{\mathrm{MDC}-4}$ compression function. For convenience, we swapped the left and right block ciphers of the second $f_{\mathrm{MDC}-2}$ evaluation.

$E$ requires at least $2^{3 n / 5}$ queries (asymptotically) to find a collision. His proof relies on the observation that a collision for MDC-2 implies a collision for the last two rounds of MDC-2, except for some special cases. These results on MDC-2 are summarized in Table 1; all of these results hold for the case $E_{1}$ and $E_{2}$ are different block ciphers as for the case they are the same.

The MDC-4 hash function differs from MDC-2 in the sense that its underlying compression function $f_{\text {MDC-4 }}$ makes four calls to the underlying block cipher rather than two. $f_{\text {MDC-4 }}$ is defined as two consecutive evaluations of $f_{\mathrm{MDC}-2}$, where the message inputs to the MMO executions in the second evaluation are $B$ for $E_{1}$ and $A$ for $E_{2}$. The definition of $f_{\mathrm{MDC}-4}: \mathbb{Z}_{2}^{3 n} \rightarrow \mathbb{Z}_{2}^{2 n}$ is given in Fig. 1. Knudsen and Preneel [16] showed that approximately $2^{3 n / 4}$ block cipher executions suffice to find a collision for $f_{\text {MDC-4}}$. With respect to preimage resistance, the same authors report that $2^{3 n / 2}$ calls suffice for finding a preimage for $f_{\mathrm{MDC}-4}$ and $2^{7 n / 4}$ calls result in a preimage for MDC-4. The latter result is recently improved to $2^{3 n / 2}$ by Hong and Kwon [12. These results are summarized in Table 1. In independent concurrent research, Fleischmann et al. 7, 8, analyzed the collision resistance of MDC-4. They proved that MDC-4 is collision secure up to approximately $2^{3 n / 5}$ queries. Hence, they prove that the bound of Steinberger for MDC-2 also holds for MDC-4 (in fact, numerically their bound is worse than the bound on MDC-2). A preimage security lower bound has so far never been derived. Since the introduction of the functions in [4, 25], however, the MDC-4 hash function has always been considered the more secure variant of MDC-2. Although mainly a matter of theoretical interest (given that MDC-2 is twice as fast as MDC-4), formal security lower bounds for MDC- 4 or $f_{\text {MDC-4 }}$ that confirm this longstanding claim have never been derived. In particular, for years the MDC-4 structure has not been thoroughly analyzed, which makes it impossible to classify MDC-4 among other double block length constructions known in literature (see "Related Work").

\section{Our Contributions}

In this work, we formally analyze the collision and (everywhere) preimage security of MDC-4 and its underlying $f_{\text {MDC-4 }}$ compression function of Fig. 1. We start with considering the general setting where $f_{\text {MDC-4 }}$ is built on one block cipher $E=E_{1}=E_{2}$, the single block cipher setting. Then, we consider the more constrained double block cipher setting, where MDC-4 is based on two independently distributed ciphers $E_{1}$ and $E_{2}$.

Single block cipher setting. A customary approach for proving collision and preimage resistance of a hash function is to analyze the compression function first and use a preservation result to show that the findings also apply to the full hash function. However, when the two block ciphers underlying MDC-4 are modeled as one single random block cipher $E$, collisions and preimages for $f_{\mathrm{MDC}-4}$ can be found in at most $2^{n / 2}$ and $2^{n}$ block cipher calls, respectively: one focuses on state values with the same left and right 
Table 1. Known security results for the MDC-2 and MDC-4 compression and hash functions. The security bound gives a lower bound and the attack bound gives an upper bound on the number of queries in order to find an attack. By "(triv)" we note that the bound is trivial; these bounds come from the security of the MMO construction [3], or correspond to generic attacks. The results printed in bold are derived in this work.

\begin{tabular}{|c|c|c|c|c|c|}
\hline & \multicolumn{2}{|c|}{ collision } & \multicolumn{2}{|c|}{ preimage } & \multirow{2}{*}{$\begin{array}{c}\text { ideal } \\
\text { primitives }\end{array}$} \\
\hline & security & attack & security & attack & \\
\hline$f_{\mathrm{MDC}-2}$ & $2^{n / 2}$ (triv) & $2^{n / 2}$ (triv) & $2^{n}$ (triv) & $2^{n}$ (triv) & \multirow{2}{*}{$E$ or $\left(E_{1}, E_{2}\right)$} \\
\hline MDC-2 & $2^{3 n / 5} 28$ & $2^{n} / n$ & $2^{n}$ (triv) & $2^{n}[15]$ & \\
\hline$f_{\mathrm{MDC}-4}$ & $2^{n / 2}$ (triv) & $2^{n / 2}$ (triv) & $2^{n}$ (triv) & $2^{n}$ (triv) & \multirow{2}{*}{$E$} \\
\hline MDC-4 & $2^{5 n / 8}$ & $2^{n}$ (triv) & $2^{n}$ (triv) & $2^{\mathrm{n}}$ & \\
\hline$f_{\mathrm{MDC}-4}$ & $2^{5 n / 8}$ & $2^{3 n / 4} \quad 16$ & $2^{5 n / 4}$ & $2^{3 n / 2} 16$ & \multirow{2}{*}{$\left(E_{1}, E_{2}\right)$} \\
\hline MDC-4 & $2^{5 n / 8}$ & $2^{n}($ triv $)$ & $2^{5 n / 4}$ & $2^{3 n / 2} \quad 12$ & \\
\hline
\end{tabular}

half (for Fig. 1 1 this means $A=B$ and $Y=Z$, and consequently $S=T, U=V$, and $W=X$ ), and the security boils down to the security of two subsequent MMO evaluations. For the preimage resistance, these type of target images (with $Y=Z$ ) can be considered as weak images, these give the adversary significantly more power. In any case, the security preservation approach does not help us out here, and instead we consider the security of the MDC-4 hash function design directly.

Starting with collision resistance, we formally prove that any adversary with query access to $E$, modeled as an ideal cipher, needs at least $2^{5 n / 8}$ queries (asymptotically) to find a collision for MDC-4. This is beyond the collision bound $2^{3 n / 5}$ on MDC-2 by Steinberger 28] and on MDC- 4 concurrently derived by Fleischmann et al. [7, 8]. The proof consists of two parts: given that the initial value of MDC4 consists of two different halves, we prove that all intermediate state values of a MDC-4 evaluation consist of different halves, except with a small probability. Then, it suffices to analyze collision resistance of $f_{\mathrm{MDC}-4}$ restricted to state values with different left and right halves, which we prove up to at least $2^{5 n / 8}$ queries. At the first glance, as $f_{\mathrm{MDC}-4}$ roughly consists of two evaluations of $f_{\mathrm{MDC}-2}$, one is inclined to say the results of Steinberger [28] directly carry over. However, this is not true due to the differences at the second $f_{\mathrm{MDC}-2}$ evaluation where the inputs are swapped and the message inputs differ for the left and right cipher. Instead, we conduct a new collision resistance proof for $f_{\mathrm{MDC}-4}$, and although it shows some similarities with the proof of Steinberger, it differs in many aspects and uses several new ideas to facilitate the analysis.

For (everywhere) preimage resistance, we derive a more surprising result, namely that if the target image $(Y, Z)$ satisfies $Y=Z$, a preimage for the MDC-4 hash function can be found in approximately $2^{n}$ queries. The attack resembles ideas from the preimage attack on MDC-2 by Knudsen et al. [15] and from above-described preimage attack on $f_{\mathrm{MDC}-4}$ in the single block cipher setting. We stress that the attack does not apply to the original MDC-4 design due to the domain separation by the functions $v$ and $w$. On the other hand, if the target image satisfies $Y \neq Z$, we prove that any adversary requires at least $2^{5 n / 4}$ queries (asymptotically) to find a preimage for $f_{\mathrm{MDC}-4}$ or MDC-4, hence security beyond the birthday bound is achieved. In order to achieve security beyond $2^{n}$ queries, we employ the ideas of free queries and wish lists. These proof tools have been used before by Armknecht et al. and Lee et al. [2, 21] for compression functions based on two block cipher calls (see "Related Work"), but because MDC-4 makes four block cipher calls rather than two, and additionally the corresponding wish lists are much harder to bound, the security proof has become considerably more complex. We remark that target images with the same left and right half are rather rare, $2^{n}$ out of $2^{2 n}$ target images satisfy this property. If we had opted for preimage resistance where the challenge is randomly generated, we obtain in the single block cipher setting a security bound of approximately $2^{5 n / 4}$ queries for $f_{\mathrm{MDC}-4}$ and MDC-4, rather than the bound of $2^{n}$ queries.

The findings on MDC-4 based on one block cipher $E$ are included in Table 1 .

Double block cipher setting. As a second part of this paper, we consider the security of the MDC-4 design where the two block ciphers $E_{1}$ and $E_{2}$ are modeled as two independent ideal ciphers. We note 
that, despite its more constrained character, this model is closer to the original design due to the domain separation by the functions $v$ and $w$.

We show that in this model, any adversary with query access to $E_{1}$ and $E_{2}$, needs at least $2^{5 n / 8}$ queries (asymptotically) to find a collision for $f_{\mathrm{MDC}-4}$ and at least $2^{5 n / 4}$ queries (asymptotically) to find a preimage for $f_{\text {MDC-4 }}$. These results almost immediately follow from the results in the single block cipher setting. Note that here we do not make the restriction that the state values should consist of different halves. Because MDC-4 is a MD transform, it preserves collision and (everywhere) preimage resistance [1, which means that if the compression function satisfies these security notions, then so does the hash function. Therefore, these results for $f_{\mathrm{MDC}-4}$ directly carry over to the MDC-4 hash function.

With the $2^{5 n / 8}$ collision and $2^{5 n / 4}$ preimage security bounds we have formally confirmed the widespread belief that MDC-4 offers a higher level of security compared to MDC-2. Despite that this security gain is obtained at the price of efficiency loss, it is an interesting and important result that allows us to make a fairer comparison among the double block length hash functions and that gives us more insight in the possibilities and impossibilities of block cipher based hashing. In particular, to our knowledge this is the first time the preimage resistance of a double block length compression function design with more than two block cipher evaluations is analyzed. Given that MDC-4 is originally not constructed from a provable security point of view (but rather an efficiency point of view), more elaborate designs with more than two block cipher calls may likely offer a higher level of security; our work is a good starting point for this research direction. Although our findings improve the existing bounds on MDC-4 significantly, large gaps between the security bounds and the best known attacks remain. A more technical and elaborate analysis may result in better bounds, and it remains an interesting open problem to improve the security bounds or the generic attacks for MDC-2 and MDC-4.

\section{Related Work}

Closely related to this work are the classical double block length compression functions Abreast-DM and Tandem-DM [17] and Hirose's compression function [11, as well as the general compression function designs by Hirose [10] and Özen and Stam [26]. And in fact, these constructions all beat MDC-2 and MDC-4 with respect to collision and preimage resistance. Each of these constructions is provided with almost optimal collision security (see Fleischmann et al. 9] and Lee and Kwon [18] for Abreast-DM, see Lee et al. 20 for Tandem-DM). With respect to preimage resistance, Armknecht et al. and Lee et al. [2, 21] prove security of Abreast-DM, Tandem-DM and Hirose's compression function up to almost $2^{2 n}$ queries (as mentioned, our preimage resistance proof of MDC-4 employs some proof ideas from [2, 21]). These double block length constructions, however, all fundamentally differ from MDC-2 and MDC-4 in the sense that their underlying block ciphers have a double key size, i.e. they use a block cipher $E: \mathbb{Z}_{2}^{2 n} \times \mathbb{Z}_{2}^{n} \rightarrow \mathbb{Z}_{2}^{n}$ that clearly allows for higher compression and that renders a much stronger underlying assumption. Regarding primitives using an $n$-bit key block cipher, an interesting design is by Jetchev et al. [14], who presented a two-call function achieving $2^{2 n / 3}$ collision and $2^{n}$ preimage security. In [23], Mennink presented a class of compression functions making three block cipher calls with optimal $2^{n}$ collision security and preimage security up to $2^{3 n / 2}$ queries.

\section{Outline}

In Sect. 2, we introduce some mathematical background and the security model used in this work. The security result on the collision resistance of MDC-4 (based on one block cipher $E=E_{1}=E_{2}$ ) is given in Sect. 3, along with its formal security proof. In Sect. 4, we present our security result on the preimage resistance of MDC-4 (based on E). In Sect. 5, we discuss the implications of these results to the MDC-4 design based on two block ciphers $E_{1}, E_{2}$.

\section{Preliminaries}

For $n \in \mathbb{N}$, by $\mathbb{Z}_{2}^{n}$ we denote the set of bit strings of length $n$. For two bit strings $X, Y$, by $X \| Y$ we denote their concatenation and by $X \oplus Y$ their bitwise XOR. If $X$ is of even length, we denote by $X^{l}$ 
and $X^{r}$ its left and right halves. Throughout, we assume $n$ is even. We denote by $\operatorname{Bloc}(n)$ the set of all block ciphers $E: \mathbb{Z}_{2}^{n} \times \mathbb{Z}_{2}^{n} \rightarrow \mathbb{Z}_{2}^{n}$, where the first input corresponds to the key input.

An adversary $\mathcal{A}$ is a probabilistic algorithm with oracle access to two block ciphers $E_{1}, E_{2} \stackrel{\$}{\leftarrow} \operatorname{Bloc}(n)$ randomly sampled from $\operatorname{Bloc}(n)$ (the security model for the single block cipher setting follows after straightforward simplifications). We consider the adversary to be information-theoretic, which means that it has unbounded computational power, and that its complexity is measured by the number of queries made to its oracles. The adversary can make forward and inverse queries to $E_{1}$ and $E_{2}$. The queries are stored in a query history $\mathcal{Q}$ as elements $\left(k_{i}, K_{i}, x_{i}, y_{i}\right)$, where $i$ is the index of the query, $k_{i} \in\{1,2\}$ indicates the corresponding block cipher, $K_{i}$ is the key input, and $x_{i}$ and $y_{i}$ denote the (plaintext) input and (ciphertext) output of the block cipher. By $x_{i} \oplus y_{i}$, we define its XOR-output. The index $i$ and the parameter $k$ are omitted if they are irrelevant or clear from the context. For $q \geq 0$, by $\mathcal{Q}_{q}$ we define the query history after $q$ queries. We assume that the adversary never makes queries to which it knows the answer in advance. In this work, we consider two types of adversaries, namely one that aims at finding collisions and one that aims at finding preimages for $f_{\mathrm{MDC}-4}$.

We say that adversary $\mathcal{A}$ finds a collision for $f_{\mathrm{MDC}-4}$ if it obtains a query history $\mathcal{Q}$ that allows it to output two distinct tuples $\left(A_{1}, B_{1}, C_{1}\right),\left(A_{2}, B_{2}, C_{2}\right) \in \mathbb{Z}_{2}^{3 n}$ such that $f_{\text {MDC- } 4}\left(A_{1}, B_{1}, C_{1}\right)=$ $f_{\text {MDC-4 }}\left(A_{2}, B_{2}, C_{2}\right)$ and for which $\mathcal{Q}$ contains all block cipher queries required to compute the two evaluations of $f_{\mathrm{MDC}-4}$. We define by

$$
\operatorname{adv}_{f_{\mathrm{MDC}-4}}^{\mathrm{col}}(\mathcal{A})=\operatorname{Pr}\left(\begin{array}{r}
E_{1}, E_{2} \stackrel{\$}{\leftarrow} \operatorname{Bloc}(n),\left(A_{1}, B_{1}, C_{1}\right),\left(A_{2}, B_{2}, C_{2}\right) \leftarrow \mathcal{A}^{E_{i}, E_{i}^{-1}}: \\
\left(A_{1}, B_{1}, C_{1}\right) \neq\left(A_{2}, B_{2}, C_{2}\right), f_{\mathrm{MDC}-4}\left(A_{1}, B_{1}, C_{1}\right)=f_{\mathrm{MDC}-4}\left(A_{2}, B_{2}, C_{2}\right)
\end{array}\right)
$$

the probability that $\mathcal{A}$ succeeds in finding such query history, and define by $\mathbf{a d v}_{f_{\text {MDC- }}}^{\text {col }}(q)$ the maximum collision advantage taken over all adversaries making $q$ queries. By $\mathbf{a d v}_{f_{\mathrm{MDC}-4}}^{\mathrm{col}(\neq)}(q)$ we denote $\mathbf{a d v}_{f_{\mathrm{MDC}-4}}^{\mathrm{col}}(q)$ with the restriction that the state values $\left(A_{1}, B_{1}\right)$ and $\left(A_{2}, B_{2}\right)$ should satisfy $A_{1} \neq B_{1}$ and $A_{2} \neq B_{2}$.

With respect to preimage resistance, we opt for the notion of everywhere preimage resistance [27. This security notion intuitively guarantees preimage security for every range point. Before making queries to its oracles, the (preimage finding) adversary $\mathcal{A}$ decides on a range point $(Y, Z) \in \mathbb{Z}_{2}^{2 n}$. We say that $\mathcal{A}$ finds a preimage for $f_{\mathrm{MDC}-4}$ if it obtains a query history $\mathcal{Q}$ that allows it to output a tuple $(A, B, C) \in \mathbb{Z}_{2}^{3 n}$ such that $f_{\text {MDC- } 4}(A, B, C)=(Y, Z)$ and for which $\mathcal{Q}$ contains all block cipher queries required to compute the evaluation of $f_{\mathrm{MDC}-4}$. We define by

$$
\mathbf{a d v}_{f_{\mathrm{MDC}-4}}^{\text {epre }}(\mathcal{A})=\max _{(Y, Z) \in \mathbb{Z}_{2}^{2 n}} \operatorname{Pr}\left(\begin{array}{c}
E_{1}, E_{2} \stackrel{\$}{\leftarrow} \operatorname{Bloc}(n),(A, B, C) \leftarrow \mathcal{A}^{E_{i}, E_{i}^{-1}}(Y, Z): \\
f_{\mathrm{MDC}-4}(A, B, C)=(Y, Z)
\end{array}\right)
$$

the maximum probability that $\mathcal{A}$ succeeds in finding such query history, and define by $\mathbf{a d v}_{f_{\mathrm{MDC}-4}}^{\text {epre }}(q)$ the maximum (everywhere) preimage advantage taken over all adversaries making $q$ queries. By $\operatorname{adv}_{f_{\text {MDC- }}}^{\text {epre }(\neq)}(q)$ we denote $\mathbf{a d v}_{f_{\mathrm{MDC}-4}}^{\text {epre }}(q)$ restricted to target images $(Y, Z)$ with $Y \neq Z$.

The security definitions for the full MDC-4 hash function are defined similarly. Here, rather than tuples from $\mathbb{Z}_{2}^{3 n}$ the adversary outputs messages of arbitrary length. Throughout, we denote the initial state value of MDC-4 by $\left(F_{0}, G_{0}\right)$. In the single block cipher setting, we consider one block cipher $E$ to be generated randomly from $\operatorname{Bloc}(n)$ rather than two, and the definitions follow immediately. In the remainder of this work, it is clear from the context which of the security models we consider.

\section{Collision Resistance of MDC-4}

We derive a collision security lower bound for MDC-4 in the case the two underlying block ciphers are identical, i.e. $E=E_{1}=E_{2}$. Due to the attack on $f_{\mathrm{MDC}-4}$ (described in Sect. 1), the classical way of proving collision resistance by ways of property preservation does not work here. Therefore, the security analysis is done in a slightly different way. Recall that the attack on $f_{\mathrm{MDC}-4}$ relies on the property that the block cipher evaluations on the left and right sides of Fig. 1 can be the same. The proof is now roughly divided into two parts: we first prove that, restricted to states with different halves, $f_{\mathrm{MDC}-4}$ is collision resistant up to at least approximately $2^{5 n / 8}$ block cipher queries. Next, we show how this result can be used to prove collision resistance of the full MDC-4. 
Theorem 1. Let $n \in \mathbb{Z}_{2}^{n}$ and $q<2^{n-1}$. Let $t_{1}, t_{2}, t_{3}>0$ be any integral values. Then,

$$
\mathbf{a d v}_{f_{\mathrm{MDC}-4}}^{\mathrm{col}(\neq)}(q) \leq \frac{2\left(t_{1}+11 t_{1} t_{2}+3 t_{1} t_{2} t_{3}+12 t_{2}+4 t_{2}^{2}\right) q}{2^{n}}+\frac{2 q^{2}}{t_{1} 2^{n}}+2 \cdot 2^{n / 2}\left(\frac{2 e q}{t_{2} 2^{n / 2}}\right)^{t_{2}}+2^{n}\left(\frac{2 e q}{t_{3} 2^{n}}\right)^{t_{3}}
$$

The proof of Thm. 1 is given in Sect. 3.1. It shows similarities with the proof by Steinberger [28] of collision resistance of the MDC-2 hash function, but its structure is entirely different so as to facilitate the proof. The proof is based on using thresholds $t_{1}, t_{2}, t_{3}$, and (1) holds for any choice of these values. We elaborate on this threshold approach after Thm. 2 .

Employing this result, we now obtain the main result for the collision resistance of MDC-4 using a single block cipher $E$.

Theorem 2. Let $n \in \mathbb{Z}_{2}^{n}$ and $q<2^{n-1}$. Let $t_{1}, t_{2}, t_{3}>0$ be any integral values. Then,

$$
\mathbf{a d v}_{M D C-4}^{\mathrm{col}}(q) \leq \frac{2\left(2 t_{1}+11 t_{1} t_{2}+3 t_{1} t_{2} t_{3}+14 t_{2}+5 t_{2}^{2}\right) q}{2^{n}}+\frac{2 q^{2}}{t_{1} 2^{n}}+2 \cdot 2^{n / 2}\left(\frac{2 e q}{t_{2} 2^{n / 2}}\right)^{t_{2}}+2^{n}\left(\frac{2 e q}{t_{3} 2^{n}}\right)^{t_{3}} .
$$

The proof of Thm. 2 is given in Sect. 3.2 , and we give a brief intuition. Recall that the attack on $f_{\text {MDC-4 }}$ relies on the fact that the two halves of the evaluation can be the same. However, for a full MDC-4 iteration, the initial state value consists of two different halves, and in fact all intermediate state values consist of two different halves, except with some small probability. Now, by ways of collision resistance preservation [1, the result of Thm. 1 carries over with as additional term a bound on the probability that the adversary ends up with a state value with two the same halves.

The proof derived for Thm. 2 (similarly for Thm. 1) is based on using threshold values $t_{1}, t_{2}, t_{3}$. This is a proof approach that has for instance been employed in [20, 23, 28]. The intuitive idea of this approach is to split the "hard" event (finding collisions) into two "less hard" events, where the thresholds form a balance between the two events: for smaller values of the thresholds, one event happens with a larger probability and the other one with a smaller probability. Due to this approach, one can divide the bound of (2) into two parts. The first term forms the first part and increases for increasing parameters $t_{1}, t_{2}, t_{3}$. The remaining three terms form the second part that decreases for increasing $t_{1}, t_{2}, t_{3}$.

Clearly, for naive choices of the values the bound of (2) becomes non-informative: for instance, if $t_{1}=1$ the term $\frac{2 q^{2}}{2^{n}}$ appears and the bound indicates security up to approximately $2^{n / 2}$ queries. However, (2) holds for all integral values $t_{1}, t_{2}, t_{3}>0$, so we can equivalently state the bound as

$$
\begin{array}{r}
\operatorname{adv}_{\mathrm{MDC}-4}^{\mathrm{col}}(q) \leq \min _{t_{1}, t_{2}, t_{3}>0} \frac{2\left(2 t_{1}+11 t_{1} t_{2}+3 t_{1} t_{2} t_{3}+14 t_{2}+5 t_{2}^{2}\right) q}{2^{n}}+ \\
\frac{2 q^{2}}{t_{1} 2^{n}}+2 \cdot 2^{n / 2}\left(\frac{2 e q}{t_{2} 2^{n / 2}}\right)^{t_{2}}+2^{n}\left(\frac{2 e q}{t_{3} 2^{n}}\right)^{t_{3}},
\end{array}
$$

where $t_{1}, t_{2}, t_{3}$ may depend on $n$. For obtaining a sharp bound, we need to fine tune the integral positive parameters $t_{1}, t_{2}, t_{3}$ so that this bound is as low as possible: the trick is to take parameters $t_{1}, t_{2}, t_{3}$ minimal so that the second part of (2) still goes to 0 for $n \rightarrow \infty$. We will show that the advantage of any adversary making slightly less than $2^{5 n / 8}$ queries approaches 0 when $n$ goes to infinity. To this end, let $\varepsilon>0$ be any parameter, we consider any adversary making at most $q=2^{5 n / 8} / n^{\varepsilon}$ queries to its oracle. We set $t_{1}=2^{2 n / 8}, t_{2}=2^{n / 8}$, and $t_{3}=3$. Here, for simplicity we assume $t_{1}, t_{2}$ to be integral. If $n$ is no multiple of 8 , one sets $t_{1}, t_{2}$ to the nearest integers. Note that these parameters satisfy $t_{1}>\frac{q^{2}}{2^{n}}$, $t_{2}>\frac{q}{2^{n / 2}}$ and $t_{3}>\frac{q}{2^{n}}$, which are minimal requirements for the second part of 2 to be $<1$. Now, it is an easy exercise to verify that the bound of (2) approaches 0 for $n \rightarrow \infty$ when $q=2^{5 n / 8} / n^{\varepsilon}$ and $t_{1}, t_{2}, t_{3}$ are as specified.

Corollary 1. For any $\varepsilon>0$, we obtain $\lim _{n \rightarrow \infty} \operatorname{adv}_{M D C-4}^{\text {col }}\left(2^{5 n / 8} / n^{\varepsilon}\right)=0$.

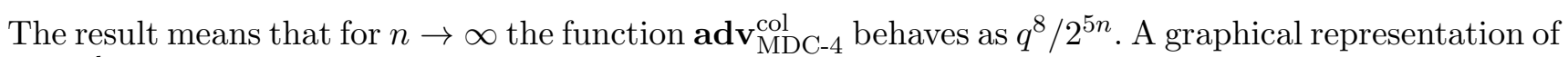
$\mathbf{a d v}_{\mathrm{MDC}-4}^{\text {col }}$ for $n=128$ is given in Fig. 2. In this graph, where we have slightly adjusted the parameters 


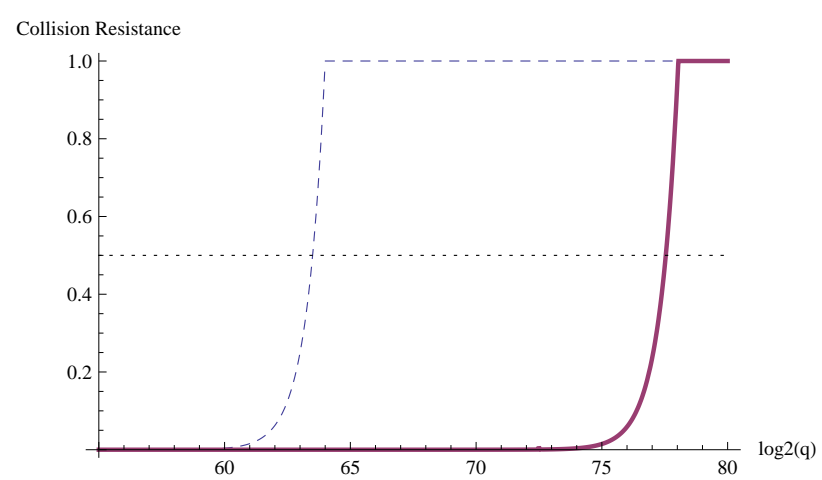

Fig. 2. The function $\operatorname{adv}_{\mathrm{MDC}-4}^{\mathrm{col}}(q)$ of 2 for $n=128$, in comparison with the trivial bound $q(q+1) / 2^{n}$ (dashed line).

$t_{1}, t_{2}$ to facilitate the analysis for smaller $n$ and smaller $q$ (the previously chosen values were set to analyze limiting behavior for $n, q$ ), we see an improvement over the best known bound and the bound independently derived in [7, 8]. For $n=128$ the collision resistance advantage hits $1 / 2$ for $\log _{2} q \approx 77.5$, which is smaller than the threshold for $q^{8} / 2^{5 n}, 79.9$. For larger values of $n$, by Cor. 1 the difference goes to 0 for $n \rightarrow \infty$.

\subsection{Proof of Thm. 1}

The collision resistance proof shows some similarities with the proof of Steinberger for MDC-2 [28], but fundamentally differs in various aspects and is as such of independent interest. In particular, due to a different and more structured case distinction we obtain a sharper bound (security up to $2^{5 n / 8}$ queries) than the bound of Steinberger for MDC-2 (security up to $2^{3 n / 5}$ queries). Also, our proof improves over the proof by Fleischmann et al. [7, 8], who basically confirmed that the $2^{3 n / 5}$ bound of MDC-2 applies to MDC-4 too.

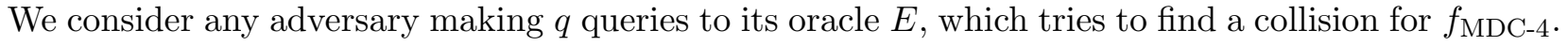
Finding a collision corresponds to obtaining a query history $\mathcal{Q}_{q}$ of size $q$ that satisfies configuration $\operatorname{col}\left(\mathcal{Q}_{q}\right)$ of Fig. 3. In other words,

$$
\mathbf{a d v}_{f_{\mathrm{MDC}-4}}^{\mathrm{col}(\neq)}(q)=\operatorname{Pr}\left(\operatorname{col}\left(\mathcal{Q}_{q}\right)\right)
$$

and we consider the probability of obtaining any query history $\mathcal{Q}_{q}$ that satisfies configuration $\operatorname{col}\left(\mathcal{Q}_{q}\right)$. Notice that in this configuration, we omit the shifting at the end: as this shifting is bijective, it does not influence the collision finding advantage. In Fig. 3, as well as in all subsequent figures in this section, we label the block ciphers as follows to uniquely identify their positions. In the left word of Fig. 3 (with inputs $\left.\left(A_{1}, B_{1}, C_{1}\right)\right)$ the block ciphers are labeled 1tl, 1tr, 1bl, 1br, for top/bottom left/right. For the right word the block ciphers are identified as $2 \mathrm{tl}, 2 \mathrm{tr}, 2 \mathrm{bl}, 2 \mathrm{br}$. In the remainder, when talking about "a query 1tl", we mean "a query that in a collision occurs at position 1tl" (and the same for the other positions). The capitalized variables in the figures may take any value, and are simply used to accentuate relations among the two words.

We need to evaluate the probability of the adversary finding a query history $\mathcal{Q}_{q}$ that satisfies configuration $\operatorname{col}\left(\mathcal{Q}_{q}\right)$ of Fig. 3. For this analysis we introduce a helping event help $\left(\mathcal{Q}_{q}\right)$. Let $t_{1}, t_{2}, t_{3}>0$ be integral. Event help $\left(\mathcal{Q}_{q}\right)$ is satisfied if either of the following sub-events help ${ }_{k}\left(\mathcal{Q}_{q}\right)(k=1, \ldots, 4)$ occurs.

$$
\begin{array}{ll}
\operatorname{help}_{1}\left(\mathcal{Q}_{q}\right): & \left|\left\{\left(K_{i}, x_{i}, y_{i}\right),\left(K_{j}, x_{j}, y_{j}\right) \in \mathcal{Q}_{q} \mid i \neq j \wedge x_{i} \oplus y_{i}=x_{j} \oplus y_{j}\right\}\right|>t_{1} ; \\
\operatorname{help}_{2}\left(\mathcal{Q}_{q}\right): & \max _{z \in \mathbb{Z}_{2}^{n / 2}}\left|\left\{\left(K_{i}, x_{i}, y_{i}\right) \in \mathcal{Q}_{q} \mid\left(x_{i} \oplus y_{i}\right)^{l}=z\right\}\right|>t_{2} ; \\
\operatorname{help}_{3}\left(\mathcal{Q}_{q}\right): & \max _{z \in \mathbb{Z}_{2}^{n / 2}}\left|\left\{\left(K_{i}, x_{i}, y_{i}\right) \in \mathcal{Q}_{q} \mid\left(x_{i} \oplus y_{i}\right)^{r}=z\right\}\right|>t_{2} ; \\
\operatorname{help}_{4}\left(\mathcal{Q}_{q}\right): & \max _{z \in \mathbb{Z}_{2}^{n}}\left|\left\{\left(K_{i}, x_{i}, y_{i}\right) \in \mathcal{Q}_{q} \mid x_{i} \oplus y_{i}=z\right\}\right|>t_{3} .
\end{array}
$$



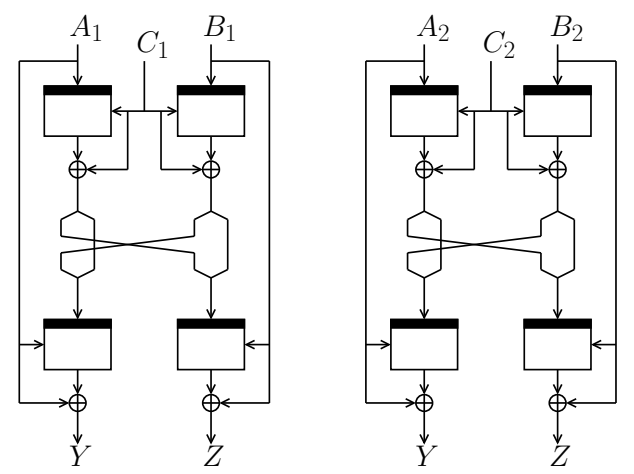

Fig. 3. Configuration $\operatorname{col}(\mathcal{Q})$. We require $\left(A_{1}, B_{1}, C_{1}\right) \neq\left(A_{2}, B_{2}, C_{2}\right), A_{1} \neq B_{1}$, and $A_{2} \neq B_{2}$.

By basic probability theory, we obtain for (3):

$$
\operatorname{Pr}\left(\operatorname{col}\left(\mathcal{Q}_{q}\right)\right) \leq \operatorname{Pr}\left(\operatorname{col}\left(\mathcal{Q}_{q}\right) \wedge \neg \operatorname{help}\left(\mathcal{Q}_{q}\right)\right)+\operatorname{Pr}\left(\operatorname{help}\left(\mathcal{Q}_{q}\right)\right) .
$$

For the analysis of the event $\operatorname{col}\left(\mathcal{Q}_{q}\right)$, it may be the case that a single query occurs at multiple positions in the configuration. Therefore, we divide $\operatorname{col}\left(\mathcal{Q}_{q}\right)$ into sub-configurations. For two distinct positions $a, b \in\{1 \mathrm{tl}, 1 \mathrm{tr}, 1 \mathrm{bl}, 1 \mathrm{br}, 2 \mathrm{tl}, 2 \mathrm{tr}, 2 \mathrm{bl}, 2 \mathrm{br}\}$ and a binary value $\alpha \in\{0,1\}$, by $a=b \equiv \alpha$ we say that the same query occurs at both positions $a$ and $b$ if and only if $\alpha=1$. Now, we define for $\alpha_{\mathrm{tl}}, \alpha_{\mathrm{tr}}, \alpha_{\mathrm{bl}}, \alpha_{\mathrm{br}} \in\{0,1\}$ the sub-configuration $\operatorname{col}_{\alpha_{\mathrm{tl}} \alpha_{\mathrm{tr}} \alpha_{\mathrm{b} \mid} \alpha_{\mathrm{br}}}(\mathcal{Q})$ as $\operatorname{col}(\mathcal{Q})$ of Fig. 3 with the restriction that

$$
1 \mathrm{tl}=2 \mathrm{tl} \equiv \alpha_{\mathrm{tl}}, \quad 1 \mathrm{tr}=2 \mathrm{tr} \equiv \alpha_{\mathrm{tr}}, \quad 1 \mathrm{bl}=2 \mathrm{bl} \equiv \alpha_{\mathrm{bl}}, \quad 1 \mathrm{br}=2 \mathrm{br} \equiv \alpha_{\mathrm{br}}
$$

Clearly,

$$
\operatorname{col}\left(\mathcal{Q}_{q}\right) \Rightarrow \bigvee_{\substack{\alpha_{\mathrm{t}}, \alpha_{\mathrm{tr}}, \alpha_{\mathrm{b}}, \alpha_{\mathrm{br}} \in\{0,1\}}} \operatorname{col}_{\alpha_{\mathrm{t} \mid} \alpha_{\mathrm{tr}} \alpha_{\mathrm{b} \mid} \alpha_{\mathrm{br}}}\left(\mathcal{Q}_{q}\right)
$$

It may be the case that the same query occurs at positions $1 \mathrm{tl}$ and $1 \mathrm{br}$ or $2 \mathrm{br}$, but as becomes clear these cases are included in the analysis. By $(35)$, we obtain the following bound on $\operatorname{adv}_{f_{\mathrm{MDC}-4}}^{\operatorname{col}(\neq)}(q)$ :

$$
\operatorname{adv}_{f_{\mathrm{MDC}-4}}^{\operatorname{col}(\neq)}(q) \leq \sum_{\substack{\alpha_{\mathrm{tl}}, \alpha_{\mathrm{tr}}, \alpha_{\mathrm{b}}, \alpha_{\mathrm{br}} \in\{0,1\}}} \operatorname{Pr}\left(\operatorname{col}_{\alpha_{\mathrm{tl}} \alpha_{\mathrm{tr}} \alpha_{\mathrm{bl}} \alpha_{\mathrm{br}}}\left(\mathcal{Q}_{q}\right) \wedge \neg \operatorname{help}\left(\mathcal{Q}_{q}\right)\right)+\operatorname{Pr}\left(\operatorname{help}\left(\mathcal{Q}_{q}\right)\right)
$$

The probabilities constituting to the sum of (6) are analyzed in Lems. 116 as further set forth in Table 2. Probability $\operatorname{Pr}\left(\operatorname{help}\left(\mathcal{Q}_{q}\right)\right)$ is analyzed in Lem. 7. In this section we only include the proof of Lem. 1 . The proofs of Lems. 22,7 are given in App. A.

Table 2. For $\alpha_{\mathrm{tl}}, \alpha_{\mathrm{tr}}, \alpha_{\mathrm{bl}}, \alpha_{\mathrm{br}} \in\{0,1\}$, the probability bound on $\operatorname{col}_{\alpha_{\mathrm{t} \mid} \alpha_{\mathrm{tr}} \alpha_{\mathrm{b} \mid} \alpha_{\mathrm{br}}}\left(\mathcal{Q}_{q}\right) \wedge \neg \operatorname{help}\left(\mathcal{Q}_{q}\right)$ (cf. (6)) is analyzed in the corresponding lemma.

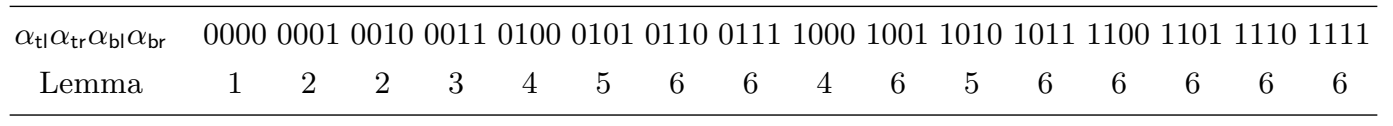

Lemma 1. $\operatorname{Pr}\left(\operatorname{col}_{0000}\left(\mathcal{Q}_{q}\right) \wedge \neg \operatorname{help}\left(\mathcal{Q}_{q}\right)\right) \leq \frac{\left(t_{1} t_{2} t_{3}+t_{1} t_{2}+2 t_{2}\right) q}{2^{n}-q}$.

Proof. A visualization of configuration $\operatorname{col}_{0000}\left(\mathcal{Q}_{q}\right)$ can be found in Fig. 4. In this figure, the queries corresponding to locations $a$ and $! a$ are required to be different, and the same for the queries at positions $(b, ! b),(c, ! c)$ and $(d, ! d)$. For the analysis of $\operatorname{col}_{0000}\left(\mathcal{Q}_{q}\right) \wedge \neg$ help $\left(\mathcal{Q}_{q}\right)$, we say that the $i$-th query $(i \in$ $\{1, \ldots, q\})$ is successful if it makes configuration $\operatorname{col}_{0000}\left(\mathcal{Q}_{i}\right)$ satisfied and $\neg$ help $\left(\mathcal{Q}_{i}\right)$ holds. Now, by 
basic probability theory, we can analyze the probability of the $i$-th query being successful, and sum over $i=1, \ldots, q$.

Let $i \in\{1, \ldots, q\}$. We will analyze the probability of the $i$-th query to be successful, i.e. to satisfy $\operatorname{col}_{0000}\left(\mathcal{Q}_{i}\right) \wedge \neg$ help $\left(\mathcal{Q}_{i}\right)$. If help $\left(\mathcal{Q}_{i}\right)$ holds, the $i$-th query can certainly not be successful, so we assume $\neg$ help $\left(\mathcal{Q}_{i}\right)$ and analyze the probability the $i$-th query makes $\operatorname{col}_{0000}\left(\mathcal{Q}_{i}\right)$ satisfied.

Without loss of generality (by symmetry), the $i$-th query occurs in the left word. It may be the case that the $i$-th query also occurs in the right word (e.g. at $2 \mathrm{br}$ ), but as becomes clear from the proof, this case is automatically included. Note that, as $A_{1} \neq B_{1}$, it can impossibly occur at (1tl, $1 \mathrm{tr}$ ) or (1bl, $\left.1 \mathrm{br}\right)$. Therefore, without loss of generality (by symmetry) it suffices to analyze the cases the query occurs at the following positions: $1 \mathrm{tl}$ only, $1 \mathrm{br}$ only, $(1 \mathrm{tl}, 1 \mathrm{br})$ only, or $(1 \mathrm{tl}, 1 \mathrm{bl})$ only. We distinguish among these four cases.

Query occurs at $1 \mathrm{tl}$ only. By $\neg$ help ${ }_{1}\left(\mathcal{Q}_{i}\right)$, there are $\leq t_{1}$ choices for queries at positions $(1 \mathrm{br}, 2 \mathrm{br})$ (we note that the query at $2 \mathrm{br}$ may equal the $i$-th query, but this does not invalidate the ongoing analysis). For any of these $\leq t_{1}$ choices, let $K_{1 \mathrm{br}}$ and $K_{2 \mathrm{br}}$ be the key inputs corresponding to positions $1 \mathrm{br}$ and $2 \mathrm{br}$. By $\neg$ help $\mathrm{p}_{2}\left(\mathcal{Q}_{i}\right)$, there are $\leq t_{2}$ choices for $2 \mathrm{tl}$. For any of these $\leq t_{1} t_{2}$ choices $2 \mathrm{tl}$, the query at position $2 \mathrm{tr}$ and consequently the query at position $2 \mathrm{bl}$ is uniquely determined (if they exist at all), and so is the XOR-output $Y$ of $2 \mathrm{bl}$. By $\neg \operatorname{hel}_{4}\left(\mathcal{Q}_{i}\right)$, there are $\leq t_{3}$ choices for $1 \mathrm{bl}$. For any of these $\leq t_{1} t_{2} t_{3}$ choices $1 \mathrm{bl}$, let $K_{1 \mathrm{bl}}$ be the key input corresponding to position $1 \mathrm{bl}$. The $i$-th query is successful only if its XOR-output equals $K_{1 \mathrm{br}}^{l} \| K_{1 \mathrm{~b}}^{r}$, which happens with probability at most $\frac{1}{2^{n}-q}$. The total probability is at most $\frac{t_{1} t_{2} t_{3}}{2^{n}-q}$.

Query occurs at $1 \mathrm{br}$ only. By $\neg$ help $\mathrm{p}_{1}\left(\mathcal{Q}_{i}\right)$, there are $\leq t_{1}$ choices for $(1 \mathrm{bl}, 2 \mathrm{bl})$. For any of these $\leq t_{1}$ choices, let $K_{2 \mathrm{~b}}$ be the key input corresponding to position $2 \mathrm{bl}$. By $\neg$ help ${ }_{2}\left(\mathcal{Q}_{i}\right)$, there are $\leq t_{2}$ choices for $2 \mathrm{tr}$. For any of these $\leq t_{1} t_{2}$ choices $2 \mathrm{tr}$, the query at position $2 \mathrm{tl}$ and consequently the query at position $2 \mathrm{br}$ is uniquely determined, and so is the XOR-output $Z$ of $2 \mathrm{br}$. The $i$-th query is successful only if its XOR-output equals this value $Z$, which happens with probability at most $\frac{1}{2^{n}-q}$. The total success probability is at most $\frac{t_{1} t_{2}}{2^{n}-q}$.

Query occurs at $1 \mathrm{tl}$ and $1 \mathrm{br}$ only. Let $K$ be the key input for the $i$-th query. As the query occurs at both positions, we require $K^{l}=Z^{l}$, which fixes $Z^{l}$. By $\neg$ help $p_{2}\left(\mathcal{Q}_{i}\right)$, there are $\leq t_{2}$ choices for $2 \mathrm{br}$. For any of these $\leq t_{2}$ choices $2 \mathrm{br}$, we obtain a different $Z$. The $i$-th query is successful only if its XOR-output equals this value $Z$, which happens with probability at most $\frac{1}{2^{n}-q}$. The total success probability is at most $\frac{t_{2}}{2^{n}-q}$.

Query occurs at $1 \mathrm{tl}$ and $1 \mathrm{bl}$ only. Let $K$ be the key input for the $i$-th query. As the query occurs at both positions, we require $K^{r}=Y^{r}$, which fixes $Y^{r}$. By $\neg$ help $p_{3}\left(\mathcal{Q}_{i}\right)$, there are $\leq t_{2}$ choices for $2 \mathrm{bl}$. For any of these $\leq t_{2}$ choices $2 \mathrm{bl}$, we obtain a different $Y$. The $i$-th query is successful only if its XOR-output equals this value $Y$, which happens with probability at most $\frac{1}{2^{n}-q}$. The total success probability is at most $\frac{t_{2}}{2^{n}-q}$.

The $i$-th query is successful with probability at most $\frac{t_{1} t_{2} t_{3}+t_{1} t_{2}+2 t_{2}}{2^{n}-q}$. The claimed bound is obtained by summing over $i=1, \ldots, q$.

Lemma 2. $\operatorname{Pr}\left(\operatorname{col}_{\alpha_{\mathrm{t} \mid} \alpha_{\mathrm{tr}} \alpha_{\mathrm{b} \mid} \alpha_{\mathrm{br}}}\left(\mathcal{Q}_{q}\right) \wedge \neg \operatorname{help}\left(\mathcal{Q}_{q}\right)\right) \leq \frac{\left(2 t_{1} t_{2}+2 t_{2}+t_{2}^{2}\right) q}{2^{n}-q}$ for $\alpha_{\mathrm{t} \mid} \alpha_{\mathrm{tr}} \alpha_{\mathrm{b} \mid} \alpha_{\mathrm{br}} \in\{0001,0010\}$.

Lemma 3. $\operatorname{Pr}\left(\operatorname{col}_{0011}\left(\mathcal{Q}_{q}\right) \wedge \neg \operatorname{help}\left(\mathcal{Q}_{q}\right)\right) \leq \frac{t_{1} q}{2^{n}-q}$.

Lemma 4. $\operatorname{Pr}\left(\operatorname{col}_{\alpha_{\mathrm{t} \mid} \alpha_{\mathrm{tr}} \alpha_{\mathrm{b} \mid} \alpha_{\mathrm{br}}}\left(\mathcal{Q}_{q}\right) \wedge \neg \operatorname{help}\left(\mathcal{Q}_{q}\right)\right) \leq \frac{\left(t_{1} t_{2} t_{3}+2 t_{1} t_{2}+2 t_{2}\right) q}{2^{n}-q}$ for $\alpha_{\mathrm{t} \mid} \alpha_{\mathrm{tr}} \alpha_{\mathrm{b} \mid} \alpha_{\mathrm{br}} \in\{0100,1000\}$.

Lemma 5. $\operatorname{Pr}\left(\operatorname{col}_{\alpha_{\mathrm{t} \mid} \alpha_{\mathrm{tr}} \alpha_{\mathrm{b} \mid} \alpha_{\mathrm{br}}}\left(\mathcal{Q}_{q}\right) \wedge \neg \operatorname{help}\left(\mathcal{Q}_{q}\right)\right) \leq \frac{\left(t_{1} t_{2}+t_{2}+t_{2}^{2}\right) q}{2^{n}-q}$ for $\alpha_{\mathrm{t} \mid} \alpha_{\mathrm{tr}} \alpha_{\mathrm{b} \mid} \alpha_{\mathrm{br}} \in\{0101,1010\}$.

Lemma 6. $\operatorname{Pr}\left(\operatorname{col}_{\alpha_{\mathrm{t} \mid} \alpha_{\mathrm{tr}} \alpha_{\mathrm{b} \mid} \alpha_{\mathrm{br}}}\left(\mathcal{Q}_{q}\right) \wedge \neg\right.$ help $\left.\left(\mathcal{Q}_{q}\right)\right)=0$ for $\alpha_{\mathrm{t} \mid} \alpha_{\mathrm{tr}} \alpha_{\mathrm{b} \mid} \alpha_{\mathrm{br}} \in\{11 * *, 1 * * 1, * 11 *\}$.

Lemma 7. $\operatorname{Pr}\left(\operatorname{help}\left(\mathcal{Q}_{q}\right)\right) \leq \frac{q^{2}}{t_{1}\left(2^{n}-q\right)}+2 \cdot 2^{n / 2}\left(\frac{e q 2^{n / 2}}{t_{2}\left(2^{n}-q\right)}\right)^{t_{2}}+2^{n}\left(\frac{e q}{t_{3}\left(2^{n}-q\right)}\right)^{t_{3}}$. 

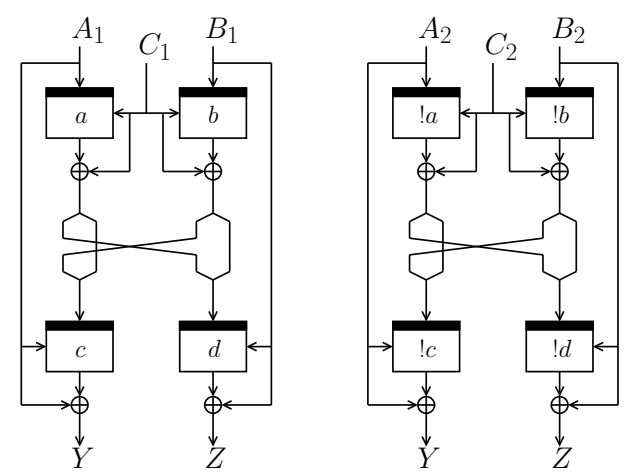

Fig. 4. Configuration $\operatorname{col}_{0000}(\mathcal{Q})$ of Lem. 1. We require $\left(A_{1}, B_{1}, C_{1}\right) \neq\left(A_{2}, B_{2}, C_{2}\right), A_{1} \neq B_{1}$, and $A_{2} \neq B_{2}$.

We are ready to finish the proof of Thm. 1. Lemmas $1-7$ imply for $\operatorname{adv}_{f_{\mathrm{MDC}-4}}^{\operatorname{col}(\neq)}(q)$ of 6 :

$$
\begin{array}{r}
\mathbf{a d v}_{f_{\mathrm{MDC}-4}}^{\operatorname{col}(\neq)}(q) \leq \frac{\left(t_{1}+11 t_{1} t_{2}+3 t_{1} t_{2} t_{3}+12 t_{2}+4 t_{2}^{2}\right) q}{2^{n}-q}+\frac{q^{2}}{t_{1}\left(2^{n}-q\right)}+ \\
2 \cdot 2^{n / 2}\left(\frac{e q 2^{n / 2}}{t_{2}\left(2^{n}-q\right)}\right)^{t_{2}}+2^{n}\left(\frac{e q}{t_{3}\left(2^{n}-q\right)}\right)^{t_{3}}
\end{array}
$$

where $t_{1}, t_{2}, t_{3}>0$ are integral. The result of Thm. 1 is obtained by observing that $2^{n}-q>2^{n-1}$ for $q<2^{n-1}$.

\subsection{Proof of Thm. 2}

As explained in Sect. 3 we essentially only need to consider the probability that an adversary finds an $f_{\text {MDC-4 }}$ evaluation where the state consists of two different halves and the output state consists of two the same halves. The formal treatment of this is more elaborate.

We consider any adversary making $q$ queries to its oracle $E$, which tries to find a collision for MDC-4. Denote by $\left(F_{0}, G_{0}\right)$ the initial state value of MDC-4, where $F_{0} \neq G_{0}$. Suppose the adversary finds a collision, i.e. two lists

$$
\begin{aligned}
& \left(F_{0}, G_{0}\right) \stackrel{m_{1}}{\longrightarrow}\left(F_{1}, G_{1}\right) \stackrel{m_{2}}{\longrightarrow} \cdots \stackrel{m_{k}}{\longrightarrow}\left(F_{k}, G_{k}\right), \\
& \left(F_{0}, G_{0}\right) \stackrel{m_{1}^{\prime}}{\longrightarrow}\left(F_{1}^{\prime}, G_{1}^{\prime}\right) \stackrel{m_{2}^{\prime}}{\longrightarrow} \cdots \stackrel{m_{k}^{\prime}}{\longrightarrow}\left(F_{k^{\prime}}^{\prime}, G_{k^{\prime}}^{\prime}\right)
\end{aligned}
$$

of internal state values of the two evaluations, where $k, k^{\prime} \geq 1$ and $\left(F_{k}, G_{k}\right)=\left(F_{k^{\prime}}^{\prime}, G_{k^{\prime}}^{\prime}\right)$. The collision is non-trivial if $k \neq k^{\prime}$ or if $\left(F_{i}, G_{i}, m_{i}\right) \neq\left(F_{i}^{\prime}, G_{i}^{\prime}, m_{i}^{\prime}\right)$ for some $i=1, \ldots, k=k^{\prime}$, and we consider non-trivial collisions only. If the adversary finds a collision of this form, we can distinguish between the following two cases:

(1) $F_{i} \neq G_{i}$ for all $i \in\{1, \ldots, k\}$ and $F_{i}^{\prime} \neq G_{i}^{\prime}$ for all $i \in\left\{1, \ldots, k^{\prime}\right\}$;

(2) $F_{i}=G_{i}$ for some $i \in\{1, \ldots, k\}$ or $F_{i}^{\prime}=G_{i}^{\prime}$ for some $i \in\left\{1, \ldots, k^{\prime}\right\}$.

Suppose the adversary finds a collision in case (1). This implies (by basic collision security preservation [1]) the adversary necessarily needs to obtain a query history $\mathcal{Q}_{q}$ of size $q$ that satisfies configuration $\operatorname{col}\left(\mathcal{Q}_{q}\right)$ of Fig. 3 .

On the other hand, suppose the adversary finds a collision in case (2). Without loss of generality, a state-half collision occurs in the first word. As $F_{0} \neq G_{0}$, there exists an $i$ such that $F_{i}=G_{i}$ but $F_{i-1} \neq G_{i-1}$. This means that in this case the adversary necessarily needs to obtain a query history $\mathcal{Q}_{q}$ of size $q$ that satisfies configuration statecol $\left(\mathcal{Q}_{q}\right)$ of Fig. 5. Here, $Z$ represents $F_{i}=G_{i}$ and $(A, B)$ represents $\left(F_{i-1}, G_{i-1}\right)$. As in Sect. 3.1, in this configuration we have omitted the shifting at the end. We stress that this does not harm the security analysis. We label the block ciphers $\mathrm{tl}, \ldots$, , br. 


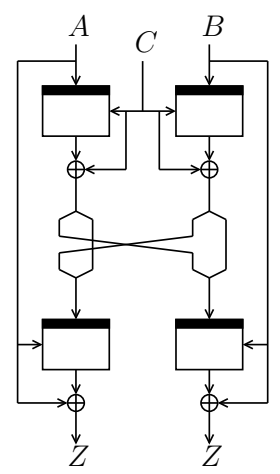

Fig. 5. Configuration statecol $(\mathcal{Q})$. We require $A \neq B$.

Concluding, we find

$$
\operatorname{adv}_{\mathrm{MDC}-4}^{\mathrm{col}}(q) \leq \operatorname{Pr}\left(\operatorname{col}\left(\mathcal{Q}_{q}\right) \vee \operatorname{statecol}\left(\mathcal{Q}_{q}\right)\right) .
$$

We employ the helping event help $\left(\mathcal{Q}_{q}\right)$ from Sect. 3.1, and obtain for $(7)$ :

$$
\begin{aligned}
\operatorname{Pr}\left(\operatorname{col}\left(\mathcal{Q}_{q}\right) \vee \text { statecol }\left(\mathcal{Q}_{q}\right)\right) \leq & \operatorname{Pr}\left(\operatorname{col}\left(\mathcal{Q}_{q}\right) \wedge \neg \text { help }\left(\mathcal{Q}_{q}\right)\right)+\operatorname{Pr}\left(\text { help }\left(\mathcal{Q}_{q}\right)\right)+ \\
& \operatorname{Pr}\left(\operatorname{statecol}\left(\mathcal{Q}_{q}\right) \wedge \neg \text { help }\left(\mathcal{Q}_{q}\right)\right) .
\end{aligned}
$$

For the first two probabilities, the proof of Thm. 1 applies. The probability bound on statecol $\left(\mathcal{Q}_{q}\right) \wedge$ $\neg$ help $\left(\mathcal{Q}_{q}\right)$ is analyzed in Lem. 8 .

Lemma 8. $\operatorname{Pr}\left(\operatorname{statecol}\left(\mathcal{Q}_{q}\right) \wedge \neg \operatorname{help}\left(\mathcal{Q}_{q}\right)\right) \leq \frac{\left(t_{1}+2 t_{2}+t_{2}^{2}\right) q}{2^{n}-q}$.

Proof. We consider configuration statecol $\left(\mathcal{Q}_{q}\right)$ of Fig. 5. The proof idea is the same as the proof of Lem. 1. Let $i \in\{1, \ldots, q\}$. As in Lem. 1, we assume $\neg$ help $\left(\mathcal{Q}_{i}\right)$ and analyze the probability the $i$-th query makes statecol $\left(\mathcal{Q}_{i}\right)$ satisfied.

Recall that the positions in Fig. 5 are simply referred to as $\mathrm{tl}, \mathrm{tr}, \mathrm{bl}, \mathrm{br}$, without a leading 1 . Without loss of generality (by symmetry), the $i$-th query occurs at the following positions: $\mathrm{tl}$ only, bl only, (tl, br) only, or (tl, bl) only. Note that, as $A \neq B$, it can impossibly occur at (tl, tr) or (bl, br).

Query occurs at $\mathrm{tl}$ only. By $\neg$ help $\mathrm{p}_{1}\left(\mathcal{Q}_{i}\right)$, there are $\leq t_{1}$ choices for $(\mathrm{bl}, \mathrm{br})$. For any of these $\leq t_{1}$ choices, let $K_{\mathrm{bl}}$ and $K_{\mathrm{br}}$ be the key inputs corresponding to positions $\mathrm{bl}$ and br. The $i$-th query is successful only if its XOR-output equals $K_{\mathrm{br}}^{l} \| K_{\mathrm{b}}^{r}$, which happens with probability at most $\frac{1}{2^{n}-q}$. The total success probability is at most $\frac{t_{1}}{2^{n}-q}$.

Query occurs at bl only. Let $K$ be the key input for the $i$-th query. By $\neg$ help $p_{2}\left(\mathcal{Q}_{i}\right)$ and $\neg$ help ${ }_{3}\left(\mathcal{Q}_{i}\right)$, there are $\leq t_{2}$ choices for $\mathrm{tl}$ and $\leq t_{2}$ choices for tr. For any of these $\leq t_{2}^{2}$ choices, the query at position br is uniquely determined, and so is the XOR-output $Z$ of br. The $i$-th query is successful only if its XORoutput equals this value $Z$, which happens with probability at most $\frac{1}{2^{n}-q}$. The total success probability is at most $\frac{t_{2}^{2}}{2^{n}-q}$.

Query occurs at $\mathrm{tl}$ and br only. Let $K$ be the key input for the $i$-th query. As the query occurs at both positions, we require $K^{l}=Z^{l}$, which fixes $Z^{l}$. By $\neg$ help ${ }_{2}\left(\mathcal{Q}_{i}\right)$, there are $\leq t_{2}$ choices for bl. For any of these $\leq t_{2}$ choices bl, we obtain a different $Z$. The $i$-th query is successful only if its XOR-output equals this value $Z$, which happens with probability at most $\frac{1}{2^{n}-q}$. The total success probability is at most $\frac{t_{2}}{2^{n}-q}$.

Query occurs at $\mathrm{tl}$ and $\mathrm{bl}$ only. Let $K$ be the key input for the $i$-th query. As the query occurs at both positions, we require $K^{r}=Z^{r}$, which fixes $Z^{r}$. By $\neg$ help $p_{3}\left(\mathcal{Q}_{i}\right)$, there are $\leq t_{2}$ choices for br. For any of these $\leq t_{2}$ choices $\mathrm{br}$, we obtain a different $Z$. The $i$-th query is successful only if its XOR-output equals this value $Z$, which happens with probability at most $\frac{1}{2^{n}-q}$. The total success probability is at most $\frac{t_{2}}{2^{n}-q}$.

The $i$-th query is successful with probability at most $\frac{t_{1}+2 t_{2}+t_{2}^{2}}{2^{n}-q}$. The claimed bound is obtained by summing over $i=1, \ldots, q$. 
We are ready to finish the proof of Thm. 2. The findings of Sect. 3.1 and Lem.8 imply for (8):

$$
\begin{array}{r}
\operatorname{adv}_{\text {MDC-4 }}^{\text {col }}(q) \leq \frac{\left(2 t_{1}+11 t_{1} t_{2}+3 t_{1} t_{2} t_{3}+14 t_{2}+5 t_{2}^{2}\right) q}{2^{n}-q}+\frac{\left(t_{1}+2 t_{2}+t_{2}^{2}\right) q}{2^{n}-q}+ \\
\frac{q^{2}}{t_{1}\left(2^{n}-q\right)}+2 \cdot 2^{n / 2}\left(\frac{e q 2^{n / 2}}{t_{2}\left(2^{n}-q\right)}\right)^{t_{2}}+2^{n}\left(\frac{e q}{t_{3}\left(2^{n}-q\right)}\right)^{t_{3}},
\end{array}
$$

where $t_{1}, t_{2}, t_{3}>0$ are integral. The result of Thm. 2 is obtained by observing that $2^{n}-q>2^{n-1}$ for $q<2^{n-1}$.

\section{Preimage Resistance of MDC-4}

We analyze the preimage security of MDC-4 in the case the two underlying block ciphers are identical, i.e. $E=E_{1}=E_{2}$. Let $(Y, Z)$ be the target image. We distinguish between $Y=Z$ and $Y \neq Z$.

$\boldsymbol{Y}=\boldsymbol{Z}$. If the two halves of the image are the same, a preimage for the compression function $f_{\mathrm{MDC}-4}$ can be found in about $2^{n}$ queries (cf. Sect. 1): one focuses on preimages with the same left and right halves $A=B$, in which case it suffices to find $A, C$ such that

$$
E(E(A, C) \oplus C, A) \oplus A=Y=Z .
$$

We demonstrate that this weakness propagates through the iteration of the MDC-4 hash function, resulting in an everywhere preimage attack for the MDC-4 hash function in $2^{n}$ queries (on average). We recall that everywhere preimage resistance is defined as the maximum advantage over all images, thus including the weak images consisting of two identical halves. If we had opted for preimage resistance where the challenge is randomly generated, this preimage attack succeeds only with small probability as $2^{n}$ out of $2^{2 n}$ target images are weak.

The attack uses ideas from Knudsen et al. [15] to find preimages for MDC-2. It is a meet-in-themiddle attack and at a high level works as follows. First, one constructs a tree with $2^{n}$ leaves with root $(Y, Z)$. The edges in this tree correspond to evaluations of $f_{\mathrm{MDC}-4}$. In the general case, the construction of this tree requires the adversary to find approximately $2^{n+1}$ preimages, but as turns out for $f_{\mathrm{MDC}-4}$ the workload is significantly lower. Then, starting from the initial value $\left(F_{0}, G_{0}\right)$, one varies the message input $C$ to hit any of the $2^{n}$ leaves. In more detail, the attack works as follows:

1. Fix any $m_{0}, m_{1} \in \mathbb{Z}_{2}^{n}$ such that $X \| m_{b}$ is a correct padding for any $X \in \mathbb{Z}_{2}^{n \cdot n}$ and $\left.b \in\{0,1\}\right|^{2}$

2. For $b=0,1$ operate as follows. For any $A \in \mathbb{Z}_{2}^{n}$ query $V \leftarrow E\left(A, m_{b}\right)$ and $W \leftarrow E\left(V \oplus m_{b}, A\right)$. These queries correspond to the evaluation $f_{\mathrm{MDC}-4}\left(A, A, m_{b}\right)=(W \oplus A, W \oplus A)$. Add the input-output tuple $((A, A) ;(W \oplus A, W \oplus A))$ to a list $L_{b}$;

3. Let $(Z, Z)$ be the target image. On average, this item occurs once in each list $L_{0}, L_{1}$, which results in two $f_{\text {MDC-4 }}$ preimages for $(Z, Z)$. It may result in more than two $f_{\text {MDC-4 }}$ preimages if $(Z, Z)$ occurs multiple times in one of the lists. The same procedure can be iteratively executed for all resulting preimages, until a tree of approximately $2^{n}$ leaves is formed, with from each leave a path of $n$ edges to $(Z, Z) 3^{3}$

4. Starting from initial value $\left(F_{0}, G_{0}\right)$, vary $m$ until $f_{\mathrm{MDC}-4}\left(F_{0}, G_{0}, m\right)$ hits any of the $2^{n}$ leaves.

Step 1 requires $2^{n+2}$ block cipher queries. Step 4 is a brute force attack and requires approximately $4 \cdot\left(2^{2 n} / 2^{n}\right)$ evaluations of $E$. In total, this attack requires approximately $2^{n+3}$ queries. The attack has time and space complexity $O\left(2^{n}\right)$.

$\boldsymbol{Y} \neq \boldsymbol{Z}$. For the everywhere preimage resistance of the $f_{\mathrm{MDC}-4}$ compression function of Fig. 1 with the restriction that $Y \neq Z$, we derive the following results. The findings directly carry over to MDC-4 as it is a MD transform, which preserves everywhere preimage resistance [1].

\footnotetext{
${ }^{2}$ We assume the padding takes less than $n$ bits.

${ }^{3}$ Due to collisions in the lists $L_{0}, L_{1}$, the amount of $2^{n}$ will usually not be reached. Elaborate statistical analysis shows that the average number of leaves at distance $n$ from the $\operatorname{root}(Z, Z)$ varies between $2^{n-1}$ and $2^{n}$.
} 


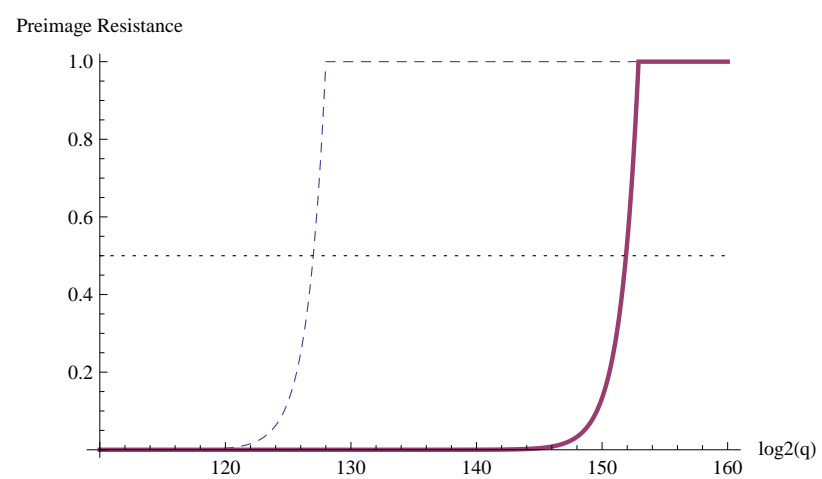

Fig. 6. The function $\operatorname{adv}_{f_{\mathrm{MDC}-4}}^{\text {epre }(\neq)}(q)$ of 99 for $n=128$, in comparison with the best known bound $q / 2^{n}$ (dashed line).

Theorem 3. Let $n \in \mathbb{Z}_{2}^{n}$. Let $t_{1}, t_{2}>0$ be any integral values with $t_{1} \leq q$. Then, provided the image $(Y, Z)$ satisfies $Y \neq Z$,

$$
\begin{aligned}
\mathbf{a d v}_{f_{\mathrm{MDC}-4}}^{\mathrm{epre}(\neq)}(q) & \leq \frac{4 t_{2}^{3}+4 t_{1} t_{2}+20+4 \cdot 2^{n / 2}}{2^{n}}+\frac{16 t_{1} t_{2}+24 t_{1} t_{2} 2^{n / 2}}{2^{2 n}}+\frac{64 q}{2^{3 n}}+ \\
2 & \cdot 2^{n / 2}\left(\frac{4 e q}{t_{1} 2^{n / 2}}\right)^{t_{1} / 2}+\frac{4 q}{2^{n / 2}}\left(\frac{8 e q}{t_{1} 2^{n / 2}}\right)^{\frac{t_{1} 2^{n}}{4 q}}+2^{n}\left(\frac{4 e q}{t_{2} 2^{n}}\right)^{t_{2} / 2}+2 q\left(\frac{8 e q}{t_{2} 2^{n}}\right)^{\frac{t_{2} 2^{n}}{4 q}} .
\end{aligned}
$$

The proof of Thm. 3 is given in Sect.4.1. It employs ideas of the preimage resistance proof by Armknecht et al. 2] and Lee et al. [19, 21] for double block length compression functions, namely the issuance of free queries and the usage of wish lists. However, the analysis has become considerably more complex because the MDC-4 compression function uses four block ciphers rather than two, and consequently the derivation of bounds on the sizes of the wish lists has become more elaborate.

The bound of $(9)$ can be analyzed in a similar manner as is done in Sect. 3 , and we skip the details. Let $\varepsilon>0$ be any parameter, we consider any adversary making at most $q=2^{5 n / 4} / n^{\varepsilon}$ queries to its oracle. We set $t_{1}=2^{3 n / 4}$ and $t_{2}=2^{n / 4} / n^{\varepsilon / 2}$. Again, $t_{1}, t_{2}$ are assumed to be integral. Note that for interesting values of $\varepsilon$, we have $t_{1} \leq q$ as desired. As before, it immediately follows that the bound of (9) approaches 0 for $n \rightarrow \infty$ when $q=2^{5 n / 4} / n^{\varepsilon}$ and $t_{1}, t_{2}$ are as specified.

Corollary 2. For any $\varepsilon>0$, we obtain $\lim _{n \rightarrow \infty} \mathbf{a d v}_{f_{\mathrm{MDC}-4}}^{\text {epre }(\neq)}\left(2^{5 n / 4} / n^{\varepsilon}\right)=0$.

The result means that for $n \rightarrow \infty$ the function $\mathbf{a d v}_{f_{\text {MDC-4 }}}^{\text {epre }(\neq)}$ (as well as $\mathbf{a d v}_{\text {MDC-4 }}^{\text {epre }(\neq)}$ by preimage resistance preservation) behaves as $q^{4} / 2^{5 n}$. a graphical representation of $\mathbf{a d v}_{f_{\mathrm{MDC}-4}}^{\text {epre }(\neq)}$ for $n=128$ is given in Fig. 6 . As in the case of Sect. 3, we have slightly adjusted the parameters $t_{1}, t_{2}$ to facilitate the analysis for smaller $n$ and smaller $q$. For $n=128$ the preimage resistance advantage hits $1 / 2$ for $\log _{2} q \approx 151.9$. Also in this case, the gap between this value and threshold for $q^{4} / 2^{5 n}, 159.75$, is caused by the choice for small $n$. By Cor. 2 the difference goes to 0 for $n \rightarrow \infty$.

\subsection{Proof of Thm. 3}

We consider any adversary making $q$ queries to its oracle $E$, which tries to find a preimage for $f_{\mathrm{MDC}-4}$. Let $(Y, Z) \in \mathbb{Z}_{2}^{2 n}$ be the point to invert, chosen by the adversary prior to making any query. Finding a preimage for $(Y, Z)$ corresponds to obtaining a query history $\mathcal{Q}_{q}$ of size $q$ that satisfies configuration $\operatorname{pre}\left(\mathcal{Q}_{q}\right)$ of Fig. 7. In other words,

$$
\operatorname{adv}_{f_{\mathrm{MDC}-4}}^{\mathrm{epre}(\neq)}(q)=\operatorname{Pr}\left(\operatorname{pre}\left(\mathcal{Q}_{q}\right)\right)
$$

and we consider the probability of obtaining any query history $\mathcal{Q}_{q}$ that satisfies configuration $\operatorname{pre}\left(\mathcal{Q}_{q}\right)$. As is done in Sect. 3.1, we again omit the bijective shifting at the end as it does not influence the preimage security. We use the same convention for the figures as is used in Sect. 3.1, with the difference 
that in Fig. 7 the variables $Y, Z$ are underlined to denote that these are fixed. As we only consider one word (rather than two, in Sect. 3.1), we label the block ciphers simply as tl, tr, bl, br for top/bottom left/right.

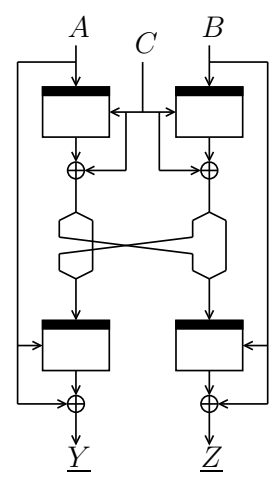

Fig. 7. Configuration pre $(\mathcal{Q})$. We have $Y \neq Z$.

The analysis in this section relies on the issuance of free super queries [2, 19, 21]. If the adversary has made $2^{n-1}$ queries to $E$ under the same key, it will receive the remaining $2^{n-1}$ queries for this key for free. As in [2, 21], we call this query a super query. Formally, these free queries can be modeled as queries the adversary is forced to make, but at no charge. For convenience, we use $\mathcal{Q}_{q}$ to denote the query history after $q$ normal queries. This query history thus contains all normal queries plus all super queries made so far. A super query is a set of $2^{n-1}$ single queries, and any query in the query history is either a normal query or a part of a super query, but not both. Notice that the adversary needs $2^{n-1}$ queries as preparatory work to enforce a super query. As the adversary makes at most $q$ queries, at most $q / 2^{n-1}$ super queries will occur.

For the analysis of $\operatorname{pre}\left(\mathcal{Q}_{q}\right)$, we introduce a helping event help $\left(\mathcal{Q}_{q}\right)$. Let $t_{1}, t_{2}>0$ be integral. Event $\operatorname{help}\left(\mathcal{Q}_{q}\right)$ is satisfied if either of the following sub-events help ${ }_{k}\left(\mathcal{Q}_{q}\right)(k=1,2,3)$ occurs.

$$
\begin{array}{ll}
\operatorname{help}_{1}\left(\mathcal{Q}_{q}\right): & \max _{z \in \mathbb{Z}_{2}^{n / 2}}\left|\left\{\left(K_{i}, x_{i}, y_{i}\right) \in \mathcal{Q}_{q} \mid\left(x_{i} \oplus y_{i}\right)^{l}=z\right\}\right|>t_{1} ; \\
\operatorname{help}_{2}\left(\mathcal{Q}_{q}\right): & \max _{z \in \mathbb{Z}_{2}^{n / 2}}\left|\left\{\left(K_{i}, x_{i}, y_{i}\right) \in \mathcal{Q}_{q} \mid\left(x_{i} \oplus y_{i}\right)^{r}=z\right\}\right|>t_{1} ; \\
\operatorname{help}_{3}\left(\mathcal{Q}_{q}\right): & \max _{z \in \mathbb{Z}_{2}^{n}}\left|\left\{\left(K_{i}, x_{i}, y_{i}\right) \in \mathcal{Q}_{q} \mid x_{i} \oplus y_{i}=z\right\}\right|>t_{2} .
\end{array}
$$

These helping events are the same as the ones used in the proof of collision resistance in Sect. 3.1. but are reintroduced for simplicity. Note that help $\mathrm{p}_{3}\left(\mathcal{Q}_{q}\right)$ particularly covers the values $Y, Z$ as XOR-outputs. By basic probability theory, we obtain for $(10)$ :

$$
\operatorname{Pr}\left(\operatorname{pre}\left(\mathcal{Q}_{q}\right)\right) \leq \operatorname{Pr}\left(\operatorname{pre}\left(\mathcal{Q}_{q}\right) \wedge \neg \operatorname{help}\left(\mathcal{Q}_{q}\right)\right)+\operatorname{Pr}\left(\operatorname{help}\left(\mathcal{Q}_{q}\right)\right) .
$$

In Lem. 9, we bound $\operatorname{Pr}\left(\operatorname{pre}\left(\mathcal{Q}_{q}\right) \wedge \neg \operatorname{help}\left(\mathcal{Q}_{q}\right)\right)$ and probability $\operatorname{Pr}\left(\operatorname{help}\left(\mathcal{Q}_{q}\right)\right)$ is analyzed in Lem. 10 The proofs are given in App. B.

Lemma 9. $\operatorname{Pr}\left(\operatorname{pre}\left(\mathcal{Q}_{q}\right) \wedge \neg \operatorname{help}\left(\mathcal{Q}_{q}\right)\right) \leq \frac{4 t_{2}^{3}+4 t_{1} t_{2}+20+4 \cdot 2^{n / 2}}{2^{n}}+\frac{16 t_{1} t_{2}+24 t_{1} t_{2} 2^{n / 2}}{2^{2 n}}+\frac{64 q}{2^{3 n}}$.

Lemma 10. Provided $t_{1} \leq q$, we have

$$
\operatorname{Pr}\left(\operatorname{help}\left(\mathcal{Q}_{q}\right)\right) \leq 2 \cdot 2^{n / 2}\left(\frac{4 e q}{t_{1} 2^{n / 2}}\right)^{t_{1} / 2}+\frac{4 q}{2^{n / 2}}\left(\frac{8 e q}{t_{1} 2^{n / 2}}\right)^{\frac{t_{1} 2^{n}}{4 q}}+2^{n}\left(\frac{4 e q}{t_{2} 2^{n}}\right)^{t_{2} / 2}+2 q\left(\frac{8 e q}{t_{2} 2^{n}}\right)^{\frac{t_{2} 2^{n}}{4 q}} .
$$

With respect to Lem. 10, we note that $\operatorname{help}_{3}(\mathcal{Q})$ is similar to the event $\operatorname{Lucky}(\mathcal{Q})$ analyzed by $\operatorname{Armknecht}$ et al. [2] and Lee et al. [21]: the only difference is that $\operatorname{hel}_{3}(\mathcal{Q})$ is required to hold for any $z \in \mathbb{Z}_{2}^{n}$. In their analysis of $\operatorname{Lucky}(\mathcal{Q}),[2,21$ make a distinction between the normal and super queries (just as we do in the proof of Lem. 10 but for the super queries their analysis is based on Markov's inequality and 
is consequently much simpler. However, because our helping events are required to hold for any $z \in \mathbb{Z}_{2}^{n}$

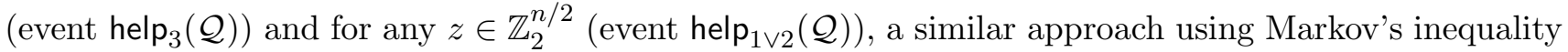
would result in a trivial bound and a more elaborate treatment was required.

The proof of Thm. 3 is finished by adding the bounds of Lems. 9 10 , as set forth in (10,11).

\section{Security of MDC-4 with Two Distinct Block Ciphers $E_{1}, E_{2}$}

We consider the collision and preimage resistance of MDC-4 in the setting where the two block ciphers $E_{1}, E_{2}$ are independently distributed. Although the analysis of MDC-4 with one block cipher is more general, the MDC-4 mode of operation with two independent block cipher is much closer to the original design due to the domain separation by the functions $v$ and $w$ (see Sect. 1). In this setting, the following results can be obtained by straightforward simplifications of the proofs of Thms. 1 and 3 .

Starting with collision resistance, we obtain the following results. They directly carry over to MDC-4 as it is a MD transform, which preserves collision resistance [1]. We stress that we pose no limitation on the state values.

Theorem 4. Let $n \in \mathbb{Z}_{2}^{n}$ and $q<2^{n-1}$. Let $t_{1}, t_{2}, t_{3}>0$ be any integral values. Then,

$$
\mathbf{a d v}_{f_{\mathrm{MDC}-4}}^{\mathrm{col}}(q) \leq \frac{2\left(t_{1}+7 t_{1} t_{2}+3 t_{1} t_{2} t_{3}+5 t_{2}+4 t_{2}^{2}\right) q}{2^{n}}+\frac{2 q^{2}}{t_{1} 2^{n}}+2 \cdot 2^{n / 2}\left(\frac{2 e q}{t_{2} 2^{n / 2}}\right)^{t_{2}}+2^{n}\left(\frac{2 e q}{t_{3} 2^{n}}\right)^{t_{3}}
$$

In the proof of Thm. 1, the restrictions $A_{1} \neq B_{1}$ and $A_{2} \neq B_{2}$ are essentially used to guarantee that the queries occurring at positions $(1 \mathrm{tl}, 1 \mathrm{tr})$ are distinct, and so are the ones at ( $1 \mathrm{bl}, 1 \mathrm{br})$. But as, regarding Thm. 4, $E_{1}, E_{2}$ are independently distributed this is directly guaranteed. The bound of Thm. 4 immediately follows by leaving out some cases in the proof of Thm. 1 ( $\operatorname{such}$ as for $\operatorname{col}_{0000}\left(\mathcal{Q}_{q}\right)$ the case that the last query appears at $(1 \mathrm{tl}, 1 \mathrm{bl}))$. By a similar reasoning as before, Cor. 11 applies to $\mathbf{a d v}_{f_{\mathrm{MDC}-4}}^{\mathrm{col}}(q)$ too.

By the same arguments, we find the following bound on the preimage resistance. Corollary 2 applies to $\mathbf{a d v}_{f_{\text {MDC- }}}^{\text {epre }}(q)$ too. The results directly carry over to MDC-4 as it is a MD transform, which preserves everywhere preimage resistance [1].

Theorem 5. Let $n \in \mathbb{Z}_{2}^{n}$. Let $t_{1}, t_{2}>0$ be any integral values with $t_{1} \leq q$. Then,

$$
\begin{array}{r}
\operatorname{adv}_{f_{\mathrm{MDC}-4}}^{\text {epre }}(q) \leq \frac{4 t_{2}^{3}+4 t_{1} t_{2}}{2^{n}}+\frac{16 t_{1} t_{2}}{2^{2 n}}+\frac{8}{2^{n}}+2 \cdot 2^{n / 2}\left(\frac{4 e q}{t_{1} 2^{n / 2}}\right)^{t_{1} / 2}+ \\
\frac{4 q}{2^{n / 2}}\left(\frac{8 e q}{t_{1} 2^{n / 2}}\right)^{\frac{t_{1} 2^{n}}{4 q}}+2^{n}\left(\frac{4 e q}{t_{2} 2^{n}}\right)^{t_{2} / 2}+2 q\left(\frac{8 e q}{t_{2} 2^{n}}\right)^{\frac{t_{2} 2^{n}}{4 q}} .
\end{array}
$$

ACKnowledgments. This work has been funded in part by the IAP Program P6/26 BCRYPT of the Belgian State (Belgian Science Policy), in part by the European Commission through the ICT program under contract ICT-2007-216676 ECRYPT II, and in part by the Research Council K.U.Leuven: GOA TENSE. The author is supported by a Ph.D. Fellowship from the Institute for the Promotion of Innovation through Science and Technology in Flanders (IWT-Vlaanderen).

\section{References}

[1] Andreeva, E., Neven, G., Preneel, B., Shrimpton, T.: Seven-property-preserving iterated hashing: ROX. In: Advances in Cryptology - ASIACRYPT 2007. Lecture Notes in Computer Science, vol. 4833, pp. 130-146. Springer, Heidelberg (2007)

[2] Armknecht, F., Fleischmann, E., Krause, M., Lee, J., Stam, M., Steinberger, J.: The preimage security of doubleblock-length compression functions. In: Advances in Cryptology - ASIACRYPT 2011. Lecture Notes in Computer Science, vol. 7073, pp. 233-251. Springer, Heidelberg (2011)

[3] Black, J., Rogaway, P., Shrimpton, T.: Black-box analysis of the block-cipher-based hash-function constructions from PGV. In: Advances in Cryptology - CRYPTO 2002. Lecture Notes in Computer Science, vol. 2442, pp. 320-335. Springer, Heidelberg (2002) 
[4] Brachtl, B., Coppersmith, D., Hyden, M., Matyas, S., Meyer, C., Oseas, J., Pilpel, S., Schilling, M.: Data Authentication Using Modification Detection Codes Based on a Public One Way Encryption Function. U.S. Patent Number 4,908,861 (March 13, 1990)

[5] Damgård, I.: A design principle for hash functions. In: Advances in Cryptology - CRYPTO '89. Lecture Notes in Computer Science, vol. 435, pp. 416-427. Springer, Heidelberg (1990)

[6] FIPS 140-2: Security Policy for IBM 'CryptoLite in C' (CLiC) (2003)

[7] Fleischmann, E., Forler, C., Lucks, S.: The collision security of MDC-4. In: Progress in Cryptology - AFRICACRYPT 2012. Lecture Notes in Computer Science, vol. 7374, pp. 252-269. Springer, Heidelberg (2012)

[8] Fleischmann, E., Forler, C., Lucks, S., Wenzel, J.: The collision security of MDC-4. Cryptology ePrint Archive, Report 2012/096 (2012), full version of [7]

[9] Fleischmann, E., Gorski, M., Lucks, S.: Security of cyclic double block length hash functions. In: IMA International Conference 2009. Lecture Notes in Computer Science, vol. 5921, pp. 153-175. Springer, Heidelberg (2009)

[10] Hirose, S.: Provably secure double-block-length hash functions in a black-box model. In: Information Security and Cryptology 2004. Lecture Notes in Computer Science, vol. 3506, pp. 330-342. Springer, Heidelberg (2005)

[11] Hirose, S.: Some plausible constructions of double-block-length hash functions. In: Fast Software Encryption 2006. Lecture Notes in Computer Science, vol. 4047, pp. 210-225. Springer, Heidelberg (2006)

[12] Hong, D., Kwon, D.: New preimage attack on MDC-4. Cryptology ePrint Archive, Report 2012/633 (2012)

[13] ISO/IEC 10118-2:2010. Information technology - Security techniques - Hash-functions - Part 2: Hash-functions using an $n$-bit block cipher (1994, revised in 2010)

[14] Jetchev, D., Özen, O., Stam, M.: Collisions are not incidental: A compression function exploiting discrete geometry. In: Theory of Cryptography Conference 2012. Lecture Notes in Computer Science, vol. 7194, pp. 303-320. SpringerVerlag, Berlin (2012)

[15] Knudsen, L., Mendel, F., Rechberger, C., Thomsen, S.: Cryptanalysis of MDC-2. In: Advances in Cryptology EUROCRYPT 2009. Lecture Notes in Computer Science, vol. 5479, pp. 106-120. Springer, Heidelberg (2009)

[16] Knudsen, L., Preneel, B.: Fast and secure hashing based on codes. In: Advances in Cryptology - CRYPTO '97. Lecture Notes in Computer Science, vol. 1294, pp. 485-498. Springer, Heidelberg (1997)

[17] Lai, X., Massey, J.: Hash function based on block ciphers. In: Advances in Cryptology - EUROCRYPT '92. Lecture Notes in Computer Science, vol. 658, pp. 55-70. Springer, Heidelberg (1992)

[18] Lee, J., Kwon, D.: The security of Abreast-DM in the ideal cipher model. Cryptology ePrint Archive, Report 2009/225 (2009)

[19] Lee, J., Stam, M., Steinberger, J.: The collision security of Tandem-DM in the ideal cipher model. Cryptology ePrint Archive, Report 2010/409 (2010), full version of [20]

[20] Lee, J., Stam, M., Steinberger, J.: The collision security of Tandem-DM in the ideal cipher model. In: Advances in Cryptology - CRYPTO 2011. Lecture Notes in Computer Science, vol. 6841, pp. 561-577. Springer, Heidelberg (2011)

[21] Lee, J., Stam, M., Steinberger, J.: The preimage security of double-block-length compression functions. Cryptology ePrint Archive, Report 2011/210 (2011)

[22] Matyas, S., Meyer, C., Oseas, J.: Generating strong one-way functions with cryptographic algorithm. IBM Techn. Disclosure Bull. 27(10A), 5658-5659 (1985)

[23] Mennink, B.: Optimal collision security in double block length hashing with single length key. In: Advances in Cryptology - ASIACRYPT 2012. Lecture Notes in Computer Science, Springer, Heidelberg (2012), to appear

[24] Merkle, R.: One way hash functions and DES. In: Advances in Cryptology - CRYPTO '89. Lecture Notes in Computer Science, vol. 435, pp. 428-446. Springer, Heidelberg (1990)

[25] Meyer, C., Schilling, M.: Secure program load with manipulation detection code. In: Proc. Securicom. pp. 111-130 (1988)

[26] Özen, O., Stam, M.: Another glance at double-length hashing. In: IMA International Conference 2009. Lecture Notes in Computer Science, vol. 5921, pp. 176-201. Springer, Heidelberg (2009)

[27] Rogaway, P., Shrimpton, T.: Cryptographic hash-function basics: Definitions, implications, and separations for preimage resistance, second-preimage resistance, and collision resistance. In: Fast Software Encryption 2004. Lecture Notes in Computer Science, vol. 3017, pp. 371-388. Springer, Heidelberg (2004)

[28] Steinberger, J.: The collision intractability of MDC-2 in the ideal-cipher model. In: Advances in Cryptology - EUROCRYPT 2007. Lecture Notes in Computer Science, vol. 4515, pp. 34-51. Springer, Heidelberg (2007)

\section{A Appendix to Sect. 3.1: Proofs of Lems. 2, 7}

In this appendix, we prove Lems. 2, 27 of Sect. 3.1. The proofs of Lems. 246 are supported by Figs. 8 . 11 , and for these figures the same convention is used as for Fig. 4. In particular, the queries corresponding to locations $a$ and $! a$ are required to be different, and the same for the queries at positions $(b, ! b)$ and $(c, ! c)$.

\section{A.1 Proof of Lem. 2}

The cases are equivalent by symmetry, and we consider $\operatorname{col}_{0001}\left(\mathcal{Q}_{q}\right)$ only. A visualization of configuration $\operatorname{col}_{0001}\left(\mathcal{Q}_{q}\right)$ can be found in Fig. 8. For the basic proof idea, we refer to the proof of Lem. 1. Let 

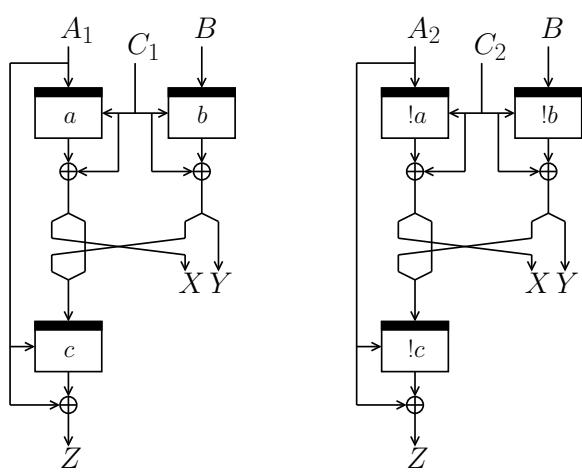

Fig. 8. Configuration $\operatorname{col}_{0001}(\mathcal{Q})$ of Lem. 2, We require $\left(A_{1}, C_{1}\right) \neq\left(A_{2}, C_{2}\right), A_{1} \neq B$, and $A_{2} \neq$ $B$.
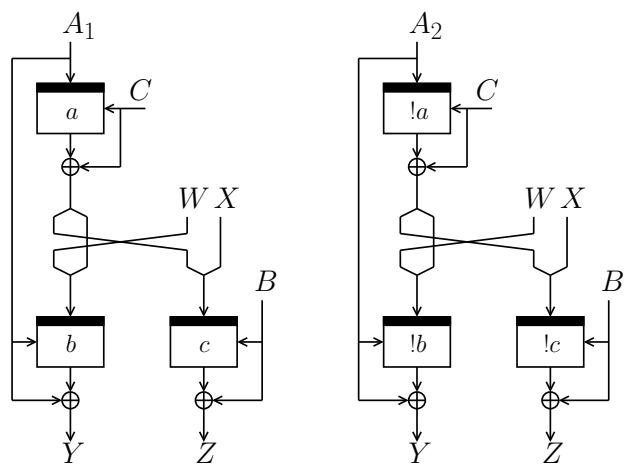

Fig. 10. Configuration $\operatorname{col}_{0100}(\mathcal{Q})$ of Lem. 4. We require $A_{1} \neq A_{2}, A_{1} \neq B$, and $A_{2} \neq B$.
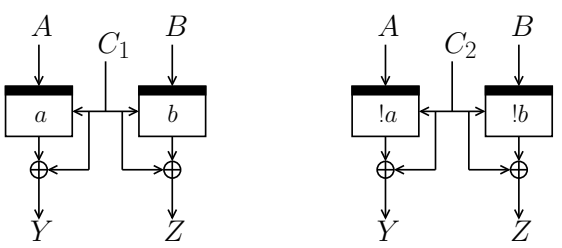

Fig. 9. Configuration $\operatorname{col}_{0011}(\mathcal{Q})$ of Lem. 3. We require $C_{1} \neq C_{2}$ and $A \neq B$.
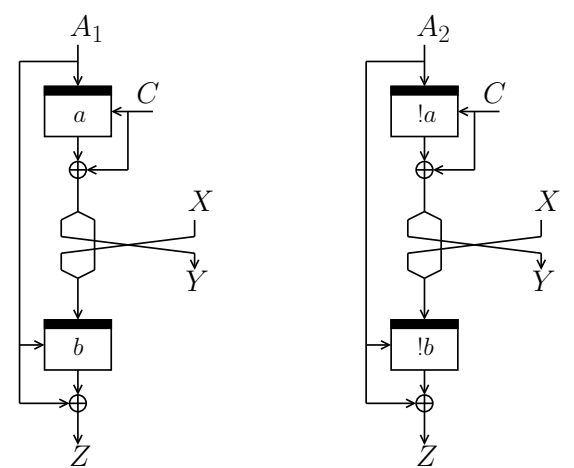

Fig. 11. Configuration $\operatorname{col}_{0101}(\mathcal{Q})$ of Lem. 5. We require $A_{1} \neq A_{2}$.

$i \in\{1, \ldots, q\}$. As in Lem. 1, we assume $\neg$ help $\left(\mathcal{Q}_{i}\right)$ and analyze the probability the $i$-th query makes $\operatorname{col}_{0001}\left(\mathcal{Q}_{i}\right)$ satisfied.

Without loss of generality (by symmetry), the $i$-th query occurs at the following positions: $1 \mathrm{tl}$ only, $1 \mathrm{tr}$ only, $1 \mathrm{bl}$ only, $(1 \mathrm{tr}, 1 \mathrm{bl})$ only, or $(1 \mathrm{tl}, 1 \mathrm{bl})$ only. We distinguish among these five cases. Note that, as $A_{1} \neq B$, it can impossibly occur at (1tl, $\left.1 \mathrm{tr}\right)$. It may be the case that the $i$-th query also occurs in the right word, but this case is automatically included.

Query occurs at $1 \mathrm{tl}$ only. By $\neg$ help $\mathrm{p}_{1}\left(\mathcal{Q}_{i}\right)$, there are $\leq t_{1}$ choices for $(1 \mathrm{bl}, 2 \mathrm{bl})$. For any of these $\leq t_{1}$ choices, let $K_{1 \mathrm{bl}}$ and $K_{2 \mathrm{bl}}$ be the key inputs corresponding to positions $1 \mathrm{bl}$ and $2 \mathrm{bl}$. By $\neg$ help $\mathrm{p}_{3}\left(\mathcal{Q}_{i}\right)$, there are $\leq t_{2}$ choices for $2 \mathrm{tl}$. For any of these $\leq t_{1} t_{2}$ choices $2 \mathrm{tl}$, we obtain a different $X$. The $i$-th query is successful only if its XOR-output equals $X \| K_{1 \mathrm{bl}}^{r}$, which happens with probability at most $\frac{1}{2^{n}-q}$. The total success probability is at most $\frac{t_{1} t_{2}}{2^{n}-q}$.

Query occurs at 1 tr only. By $\neg$ help $p_{1}\left(\mathcal{Q}_{i}\right)$, there are $\leq t_{1}$ choices for $(1 \mathrm{bl}, 2 \mathrm{bl})$. For any of these $\leq t_{1}$ choices, let $K_{1 \mathrm{bl}}$ and $K_{2 \mathrm{bl}}$ be the key inputs corresponding to positions $1 \mathrm{bl}$ and $2 \mathrm{bl}$. By $\neg$ help $\mathrm{p}_{2}\left(\mathcal{Q}_{i}\right)$, there are $\leq t_{2}$ choices for $2 \operatorname{tr}$. For any of these $\leq t_{1} t_{2}$ choices $2 \operatorname{tr}$, we obtain a different $Y$. The $i$-th query is successful only if its XOR-output equals $K_{1 \mathrm{bl}}^{l} \| Y$, which happens with probability at most $\frac{1}{2^{n}-q}$. The total success probability is at most $\frac{t_{1} t_{2}}{2^{n}-q}$.

Query occurs at 1 bl only: inverse query $x \leftarrow E^{-1}(K, y)$. By $\neg$ help $p_{3}\left(\mathcal{Q}_{i}\right)$, there are $\leq t_{2}$ choices for $1 \mathrm{tl}$. For any of these $\leq t_{2}$ choices, let $K_{1 \mathrm{tl}}$ be the key input corresponding to position $1 \mathrm{tl}$. The $i$-th query is successful only if $x=K_{1 \mathrm{tl}}$, which happens with probability at most $\frac{1}{2^{n}-q}$. The total success probability is at most $\frac{t_{2}}{2^{n}-q}$.

Query occurs at $1 \mathrm{bl}$ only: forward query $y \leftarrow E(K, x)$. By $\neg$ help ${ }_{2}\left(\mathcal{Q}_{i}\right)$, there are $\leq t_{2}$ choices for $1 \mathrm{tr}$. For any of these $\leq t_{2}$ choices $1 \mathrm{tr}$, the query at position $1 \mathrm{tl}$ is uniquely determined (it requires key input $x$ and message input $C_{1}$ defined by query $\left.1 \mathrm{tr}\right)$, and so are the strings $(B, X)$. By $\neg$ help $p_{2}\left(\mathcal{Q}_{i}\right)$, there are $\leq t_{2}$ choices for $2 \mathrm{tl}$. For any of these $\leq t_{2}^{2}$ choices $2 \mathrm{tl}$, the query at position $2 \mathrm{tr}$ is uniquely 
determined (it requires key input $B$ and message input $C_{2}$ defined by query $2 \mathrm{tl}$ ). Consequently, the query at position $2 \mathrm{br}$ is uniquely determined, and so is the XOR-output $Z$ of $2 \mathrm{br}$. The $i$-th query is successful only if its XOR-output equals this value $Z$, which happens with probability at most $\frac{1}{2^{n}-q}$. The total success probability is at most $\frac{t_{2}^{2}}{2^{n}-q}$.

Query occurs at 1 tr and $1 \mathrm{bl}$ only. Let $K$ be the key input for the $i$-th query. As the query occurs at both positions, we require $K^{l}=Z^{l}$, which fixes $Z^{l}$. By $\neg$ help $p_{2}\left(\mathcal{Q}_{i}\right)$, there are $\leq t_{2}$ choices for $2 \mathrm{bl}$. For any of these $\leq t_{2}$ choices $2 \mathrm{bl}$, we obtain a different $Z$. The $i$-th query is successful only if its XOR-output equals this value $Z$, which happens with probability at most $\frac{1}{2^{n}-q}$. The total success probability is at most $\frac{t_{2}}{2^{n}-q}$.

Query occurs at $1 \mathrm{tl}$ and $1 \mathrm{bl}$ only. Let $K$ be the key input for the $i$-th query. As the query occurs at both positions, we require $K^{r}=Z^{r}$, which fixes $Z^{r}$. By $\neg$ help $p_{3}\left(\mathcal{Q}_{i}\right)$, there are $\leq t_{2}$ choices for $2 \mathrm{bl}$. For any of these $\leq t_{2}$ choices $2 \mathrm{bl}$, we obtain a different $Z$. The $i$-th query is successful only if its XOR-output equals this value $Z$, which happens with probability at most $\frac{1}{2^{n}-q}$. The total success probability is at most $\frac{t_{2}}{2^{n}-q}$.

The $i$-th query is successful with probability at most $\frac{2 t_{1} t_{2}+2 t_{2}+t_{2}^{2}}{2^{n}-q}$. The claimed bound is obtained by summing over $i=1, \ldots, q$.

\section{A.2 Proof of Lem. 3}

A visualization of configuration $\operatorname{col}_{0011}\left(\mathcal{Q}_{q}\right)$ can be found in Fig. 9. For the basic proof idea, we refer to the proof of Lem. 1. Let $i \in\{1, \ldots, q\}$. As in Lem. 1, we assume $\neg$ help $\left(\mathcal{Q}_{i}\right)$ and analyze the probability the $i$-th query makes $\operatorname{col}_{0011}\left(\mathcal{Q}_{i}\right)$ satisfied.

Without loss of generality (by symmetry), the $i$-th query occurs at the position 1 tl only. Note that, as $A \neq B$, it can impossibly occur at $(1 \mathrm{tl}, 1 \mathrm{tr})$. It may be the case that the $i$-th query also occurs in the right word, but these cases is automatically included.

Query occurs at $1 \mathrm{tl}$. By $\neg$ help $\mathrm{p}_{1}\left(\mathcal{Q}_{i}\right)$, there are $\leq t_{1}$ choices for $(1 \mathrm{tr}, 2 \mathrm{tr})$. For any of these $\leq t_{1}$ choices, as $A$ is fixed (it equals the key input for the $i$-th query) the query at position $2 \mathrm{tl}$ is uniquely determined, and so is the XOR-output $Y$ of $2 \mathrm{tl}$. The $i$-th query is successful only if its XOR-output equals this value $Y$, which happens with probability at most $\frac{1}{2^{n}-q}$. The total success probability is at most $\frac{t_{1}}{2^{n}-q}$.

The $i$-th query is successful with probability at most $\frac{t_{1}}{2^{n}-q}$. The claimed bound is obtained by summing over $i=1, \ldots, q$.

\section{A.3 Proof of Lem. 4}

The cases are equivalent by symmetry, and we consider $\operatorname{col}_{0100}\left(\mathcal{Q}_{q}\right)$ only. A visualization of configuration $\operatorname{col}_{0100}\left(\mathcal{Q}_{q}\right)$ can be found in Fig. 10. For the basic proof idea, we refer to the proof of Lem. 1. Let $i \in\{1, \ldots, q\}$. As in Lem. 1, we assume $\neg$ help $\left(\mathcal{Q}_{i}\right)$ and analyze the probability the $i$-th query makes $\operatorname{col}_{0100}\left(\mathcal{Q}_{i}\right)$ satisfied.

Without loss of generality (by symmetry), the $i$-th query occurs at the following positions: $1 \mathrm{tl}$ only, $1 \mathrm{bl}$ only, $1 \mathrm{br}$ only, $(1 \mathrm{tl}, 1 \mathrm{br})$ only, or $(1 \mathrm{tl}, 1 \mathrm{bl})$ only. We distinguish among these five cases. Note that, as $A_{1} \neq B$, it can impossibly occur at $(1 \mathrm{bl}, 1 \mathrm{br})$. It may be the case that the $i$-th query also occurs in the right word, but this case is automatically included.

Query occurs at $1 \mathrm{tl}$ only. We note that the query at position $1 \mathrm{tr}=2 \mathrm{tr}$ is not depicted in Fig. 10 but is defined as a query $(2, B, C, W \| X \oplus C)$. By $\neg$ help $p_{1}\left(\mathcal{Q}_{i}\right)$, there are $\leq t_{1}$ choices for queries at positions $(1 \mathrm{br}, 2 \mathrm{br})$. For any of these $\leq t_{1}$ choices, let $K_{1 \mathrm{br}}$ and $K_{2 \mathrm{br}}$ be the key inputs corresponding to positions $1 \mathrm{br}$ and $2 \mathrm{br}$. By $\neg$ help $\mathrm{p}_{2}\left(\mathcal{Q}_{i}\right)$, there are $\leq t_{2}$ choices for $2 \mathrm{tl}$. For any of these $\leq t_{1} t_{2}$ choices $2 \mathrm{tl}$, the query at position $1 \mathrm{tr}=2 \mathrm{tr}$ and consequently the query at position $2 \mathrm{bl}$ is uniquely determined, and so is the XOR-output $Y$ of $2 \mathrm{bl}$. By $\neg \operatorname{hel}_{4}\left(\mathcal{Q}_{i}\right)$, there are $\leq t_{3}$ choices for $1 \mathrm{bl}$. For any of these $\leq t_{1} t_{2} t_{3}$ 
choices $1 \mathrm{bl}$, let $K_{1 \mathrm{bl}}$ be the key input corresponding to position $1 \mathrm{bl}$. The $i$-th query is successful only if its XOR-output equals $K_{1 \mathrm{br}}^{l} \| K_{1 \mathrm{bl}}^{r}$, which happens with probability at most $\frac{1}{2^{n}-q}$. The total probability is at most $\frac{t_{1} t_{2} t_{3}}{2^{n}-q}$.

Query occurs at $1 \mathrm{bl}$ only. By $\neg$ help $\mathrm{p}_{1}\left(\mathcal{Q}_{i}\right)$, there are $\leq t_{1}$ choices for $(1 \mathrm{br}, 2 \mathrm{br})$. For any of these $\leq t_{1}$ choices, let $K_{2 \mathrm{br}}$ be the key input corresponding to position $2 \mathrm{br}$. By $\neg$ help $\mathrm{p}_{2}\left(\mathcal{Q}_{i}\right)$, there are $\leq t_{2}$ choices for $2 \mathrm{tl}$. For any of these $\leq t_{1} t_{2}$ choices $2 \mathrm{tl}$, the query at position $2 \mathrm{bl}$ is uniquely determined, and so is the XOR-output $Y$ of $2 \mathrm{bl}$. The $i$-th query is successful only if its XOR-output equals this value $Y$, which happens with probability at most $\frac{1}{2^{n}-q}$. The total success probability is at most $\frac{t_{1} t_{2}}{2^{n}-q}$.

Query occurs at $1 \mathrm{br}$ only. By $\neg$ help $p_{1}\left(\mathcal{Q}_{i}\right)$, there are $\leq t_{1}$ choices for $(1 \mathrm{bl}, 2 \mathrm{bl})$. For any of these $\leq t_{1}$ choices, let $K_{2 \mathrm{bl}}$ be the key input corresponding to position $2 \mathrm{bl}$. By $\neg$ help $\mathrm{p}_{3}\left(\mathcal{Q}_{i}\right)$, there are $\leq t_{2}$ choices for $2 \mathrm{tl}$. For any of these $\leq t_{1} t_{2}$ choices $2 \mathrm{tl}$, the key input to the query at position $2 \mathrm{br}$, say $K_{2 \mathrm{br}}$, is uniquely determined. Suppose the $i$-th query is a forward query $y \leftarrow E(K, x)$ (exactly the same reasoning applies to inverse queries). If $K_{2 \mathrm{br}}=K$, the queries at positions $1 \mathrm{br}$ and $2 \mathrm{br}$ must be the same and the collision is invalid. Therefore, we assume $K_{2 \mathrm{br}} \neq K$. For the key $K_{2 \mathrm{br}}$, let $\left(K_{2 \mathrm{br}}, x_{2 \mathrm{br}}, y_{2 \mathrm{br}}\right)$ be any query in the query history. The $i$-th query makes the configuration satisfied if $x_{2 \mathrm{br}}=x$ and $x_{2 \mathrm{br}} \oplus y_{2 \mathrm{br}}=x \oplus y$, or more concretely if

$$
x_{2 \mathrm{br}}=x \text { and } y_{2 \mathrm{br}}=y .
$$

This means that, irrespectively of whether the $i$-th query is a forward or inverse query, the query at position $2 \mathrm{br}$ is uniquely determined. The $i$-th query is successful only if it satisfies (14), which happens with probability at most $\frac{1}{2^{n}-q}$. The total probability is at most $\frac{t_{1} t_{2}}{2^{n}-q}$.

Query occurs at $1 \mathrm{tl}$ and $1 \mathrm{br}$ only. Let $K$ be the key input for the $i$-th query. As the query occurs at both positions, we require $K^{l}=Z^{l}$, which fixes $Z^{l}$. By $\neg$ help $p_{2}\left(\mathcal{Q}_{i}\right)$, there are $\leq t_{2}$ choices for $2 \mathrm{br}$. For any of these $\leq t_{2}$ choices $2 \mathrm{br}$, we obtain a different $Z$. The $i$-th query is successful only if its XOR-output equals this value $Z$, which happens with probability at most $\frac{1}{2^{n}-q}$. The total success probability is at most $\frac{t_{2}}{2^{n}-q}$.

Query occurs at $1 \mathrm{tl}$ and $1 \mathrm{bl}$ only. Let $K$ be the key input for the $i$-th query. As the query occurs at both positions, we require $K^{r}=Y^{r}$, which fixes $Y^{r}$. By $\neg$ help $p_{3}\left(\mathcal{Q}_{i}\right)$, there are $\leq t_{2}$ choices for $2 \mathrm{bl}$. For any of these $\leq t_{2}$ choices $2 \mathrm{bl}$, we obtain a different $Y$. The $i$-th query is successful only if its XOR-output equals this value $Y$, which happens with probability at most $\frac{1}{2^{n}-q}$. The total success probability is at most $\frac{t_{2}}{2^{n}-q}$.

The $i$-th query is successful with probability at most $\frac{t_{1} t_{2} t_{3}+2 t_{1} t_{2}+2 t_{2}}{2^{n}-q}$. The claimed bound is obtained by summing over $i=1, \ldots, q$.

\section{A.4 Proof of Lem. 5}

The cases are equivalent by symmetry, and we consider $\operatorname{col}_{0101}\left(\mathcal{Q}_{q}\right)$ only. A visualization of configuration $\operatorname{col}_{0101}\left(\mathcal{Q}_{q}\right)$ can be found in Fig. 11. For the basic proof idea, we refer to the proof of Lem. 1. Let $i \in\{1, \ldots, q\}$. As in Lem. 1, we assume $\neg$ help $\left(\mathcal{Q}_{i}\right)$ and analyze the probability the $i$-th query makes $\operatorname{col}_{0101}\left(\mathcal{Q}_{i}\right)$ satisfied.

Without loss of generality (by symmetry), the $i$-th query occurs at the following positions: $1 \mathrm{tl}$ only, $1 \mathrm{bl}$ only, or $(1 \mathrm{tl}, 1 \mathrm{bl})$ only. We distinguish among these three cases. It may be the case that the $i$-th query also occurs in the right word, but this case is automatically included.

Query occurs at $1 \mathrm{tl}$ only. By $\neg$ help $\mathrm{p}_{1}\left(\mathcal{Q}_{i}\right)$, there are $\leq t_{1}$ choices for $(1 \mathrm{bl}, 2 \mathrm{bl})$. For any of these $\leq t_{1}$ choices, let $K_{1 \mathrm{bl}}$ and $K_{2 \mathrm{bl}}$ be the key inputs corresponding to positions $1 \mathrm{bl}$ and $2 \mathrm{bl}$. By $\neg$ help $\mathrm{p}_{3}\left(\mathcal{Q}_{i}\right)$, there are $\leq t_{2}$ choices for $2 \mathrm{tl}$. For any of these $\leq t_{1} t_{2}$ choices $2 \mathrm{tl}$, we obtain a different $Y$. The $i$-th query is successful only if its XOR-output equals $Y \| K_{1 \mathrm{bl}}^{r}$, which happens with probability at most $\frac{1}{2^{n}-q}$. The total success probability is at most $\frac{t_{1} t_{2}}{2^{n}-q}$.

Query occurs at $1 \mathrm{bl}$ only. Let $K$ be the key input for the $i$-th query. By $\neg$ help $\mathrm{p}_{3}\left(\mathcal{Q}_{i}\right)$, there are $\leq t_{2}$ choices for $1 \mathrm{tl}$. For any of these $\leq t_{2}$ choices, we obtain a different $Y$. By $\neg$ help $p_{2}\left(\mathcal{Q}_{i}\right)$, there are $\leq t_{2}$ choices for $2 \mathrm{tl}$. For any of these $\leq t_{2}^{2}$ choices $2 \mathrm{tl}$, the query at position $2 \mathrm{bl}$ is uniquely determined, and 
so is the XOR-output $Z$ of $2 \mathrm{bl}$. The $i$-th query is successful only if its XOR-output equals this value $Z$, which happens with probability at most $\frac{1}{2^{n}-q}$. The total success probability is at most $\frac{t_{2}^{2}}{2^{n}-q}$.

Query occurs at $1 \mathrm{tl}$ and $1 \mathrm{bl}$ only. Let $K$ be the key input for the $i$-th query. As the query occurs at both positions, we require $K^{r}=Z^{r}$, which fixes $Z^{r}$. By $\neg$ help $p_{3}\left(\mathcal{Q}_{i}\right)$, there are $\leq t_{2}$ choices for $2 \mathrm{bl}$. For any of these $\leq t_{2}$ choices $2 \mathrm{bl}$, we obtain a different $Z$. The $i$-th query is successful only if its XOR-output equals this value $Z$, which happens with probability at most $\frac{1}{2^{n}-q}$. The total success probability is at most $\frac{t_{2}}{2^{n}-q}$.

The $i$-th query is successful with probability at most $\frac{t_{1} t_{2}+t_{2}+t_{2}^{2}}{2^{n}-q}$. The claimed bound is obtained by summing over $i=1, \ldots, q$.

\section{A.5 Proof of Lem. 6}

If $1 \mathrm{tl}=2 \mathrm{tl}$ and $1 \mathrm{tr}=2 \mathrm{tr}$, we obtain $\left(A_{1}, B_{1}, C_{1}\right)=\left(A_{2}, B_{2}, C_{2}\right)$ in the configuration of Fig. 3 , and the collision is invalid. The same observation applies if $(1 \mathrm{tl}, 1 \mathrm{br})=(2 \mathrm{tl}, 2 \mathrm{br})$ or $(1 \mathrm{tr}, 1 \mathrm{bl})=(2 \mathrm{tr}, 2 \mathrm{bl})$.

\section{A.6 Proof of Lem. 7}

It suffices to consider the events $\operatorname{Pr}\left(\operatorname{help}_{k}\left(\mathcal{Q}_{q}\right)\right)(k=1, \ldots, 4)$ separately.

help ${ }_{1}\left(\mathcal{Q}_{q}\right)$. We copy the approach of Steinberger [28]. For $i \neq j$, the two queries $\left(K_{i}, x_{i}, y_{i}\right)$ and $\left(K_{j}, x_{j}, y_{i}\right)$ have the same XOR-output with probability at most $\frac{1}{2^{n}-q}$. Hence, the expected value $\mathrm{E}\left(x_{i} \oplus y_{i}=x_{j} \oplus y_{j}\right)$ is at most $\frac{1}{2^{n}-q}$, and consequently

$$
\mathrm{E}\left(\left|\left\{\left(K_{i}, x_{i}, y_{i}\right),\left(K_{j}, x_{j}, y_{j}\right) \in \mathcal{Q}_{q} \mid i \neq j \wedge x_{i} \oplus y_{i}=x_{j} \oplus y_{j}\right\}\right|\right) \leq \sum_{i \neq j} \frac{1}{2^{n}-q} \leq \frac{q^{2}}{2^{n}-q} .
$$

By Markov's inequality, we obtain

$$
\operatorname{Pr}\left(\operatorname{help}_{1}\left(\mathcal{Q}_{q}\right)\right) \leq \frac{q^{2}}{t_{1}\left(2^{n}-q\right)} .
$$

$\operatorname{help}_{k}\left(\mathcal{Q}_{q}\right)$ for $k \in\{2,3\}$. The cases are equivalent by symmetry, and we consider help $\mathcal{p}_{2}\left(\mathcal{Q}_{q}\right)$ only. Let $z \in \mathbb{Z}_{2}^{n / 2}$. Consider the $i$-th query $\left(K_{i}, x_{i}, y_{i}\right)$. This query makes equation $\left(x_{i} \oplus y_{i}\right)^{l}=z$ satisfied with probability at most $\frac{2^{n / 2}}{2^{n}-q}$. More than $t_{2}$ queries result in a solution with probability at most $\left(\begin{array}{c}q \\ t_{2}\end{array}\right)\left(\frac{2^{n / 2}}{2^{n}-q}\right)^{t_{2}} \leq\left(\frac{e q 2^{n / 2}}{t_{2}\left(2^{n}-q\right)}\right)^{t_{2}}$, where we use Stirling's approximation $\left(t ! \geq(t / e)^{t}\right.$ for any $\left.t\right)$. Considering any possible choice for $z$, we obtain for $k=2,3$ :

$$
\operatorname{Pr}\left(\operatorname{help}_{k}\left(\mathcal{Q}_{q}\right)\right) \leq 2^{n / 2}\left(\frac{e q 2^{n / 2}}{t_{2}\left(2^{n}-q\right)}\right)^{t_{2}} .
$$

help ${ }_{4}\left(\mathcal{Q}_{q}\right)$. A similar analysis as for help ${ }_{2}\left(\mathcal{Q}_{q}\right)$ results in the following bound:

$$
\operatorname{Pr}\left(\operatorname{help}_{4}\left(\mathcal{Q}_{q}\right)\right) \leq 2^{n}\left(\frac{e q}{t_{3}\left(2^{n}-q\right)}\right)^{t_{3}} .
$$

The claim is obtained by adding (15, 17).

\section{B Appendix to Sect. 4.1: Proofs of Lems. 9, 10}

\section{B.1 Proof of Lem. 9}

We consider the probability of the adversary finding a solution to configuration $\operatorname{pre}\left(\mathcal{Q}_{q}\right)$ of Fig. 7, in such a way that $\mathcal{Q}_{q}$ satisfies $\neg$ help $\left(\mathcal{Q}_{q}\right)$. For a set of solutions complying with configuration pre $\left(\mathcal{Q}_{q}\right)$, it may be the case that two queries are the same or belong to the same super query. We call a (normal or super) query winning if it makes the configuration satisfied for any other queries in the query history strictly before this winning query is made. We make the following distinction: 
1. The winning query contributes to exactly one position of configuration $\operatorname{pre}\left(\mathcal{Q}_{q}\right)$;

2. The winning query contributes to exactly two positions of configuration $\operatorname{pre}\left(\mathcal{Q}_{q}\right)$;

3. The winning query contributes to exactly three positions of configuration $\operatorname{pre}\left(\mathcal{Q}_{q}\right)$.

Note that a winning query cannot occur at all four positions: if this would be the case, we would have $A=B$ and thus $Y=Z$. In particular in the remainder of the proof we will use that a winning normal query cannot occur at positions (bl,br), and a winning query (normal or super) cannot contribute at positions ( $\mathrm{tl}, \mathrm{tr})$.

Case 1. In this case, the winning query may be a normal query or a super query. As in [19, 21], we make use of "wish lists" for the analysis of this case. Intuitively, a wish list is a continuously updated sequence of query tuples that would make configuration $\operatorname{pre}(\mathcal{Q})$ satisfied. During the attack of the adversary, we maintain four initially empty wish lists $\mathcal{W}_{\mathrm{tl}}, \mathcal{W}_{\mathrm{tr}}, \mathcal{W}_{\mathrm{bl}}, \mathcal{W}_{\mathrm{br}}$, corresponding to the four positions of configuration pre $(\mathcal{Q})$. If a query is made, the wish lists are updated according to the following requirements:

- If the query fits $\operatorname{pre}_{\mathrm{tl}}(\mathcal{Q})$ of Fig. 12 for any two other queries in the query history, the corresponding tuple $(A, C,(W \| X) \oplus C)$ is added to $\mathcal{W}_{\mathrm{tl}}$;

- If the query fits $\operatorname{pre}_{\operatorname{tr}}(\mathcal{Q})$ of Fig. 12 for any two other queries in the query history, the corresponding tuple $(B, C,(W \| X) \oplus C)$ is added to $\mathcal{W}_{\text {tr }}$;

- If the query fits $\operatorname{pre}_{\mathrm{bl}}(\mathcal{Q})$ of Fig. 12 for any two other queries in the query history, the corresponding tuple $(W \| X, A, Y \oplus A)$ is added to $\mathcal{W}_{\mathrm{bl}}$;

- If the query fits $\operatorname{pre}_{\mathrm{br}}(\mathcal{Q})$ of Fig. 12 for any two other queries in the query history, the corresponding tuple $(W \| X, B, Z \oplus B)$ is added to $\mathcal{W}_{\mathrm{br}}$.
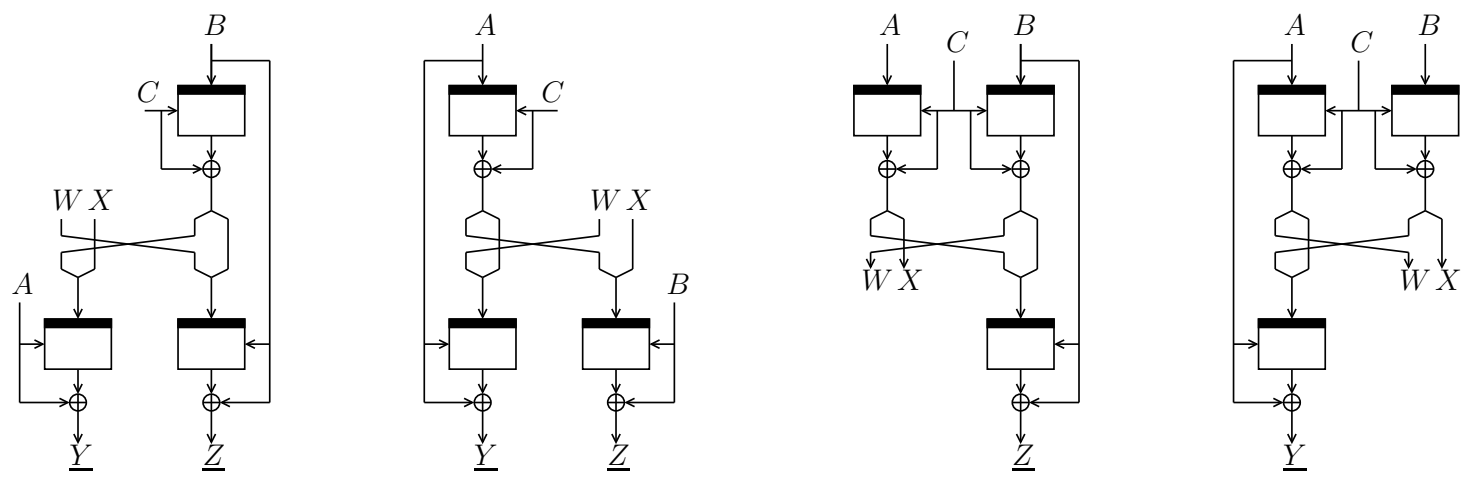

Fig. 12. From left to right: configurations $\operatorname{pre}_{\mathrm{tl}}(\mathcal{Q}), \operatorname{pre}_{\mathrm{tr}}(\mathcal{Q}), \operatorname{pre}_{\mathrm{bl}}(\mathcal{Q})$, and $\operatorname{pre}_{\mathrm{br}}(\mathcal{Q})$.

As in this case we consider the winning query to be different from all other queries made before, we can assume a query never adds itself to a wish list. It is clear that the adversary finds a preimage for MDC-4 (in this case) only if it makes a query that is already a member of any of the wish lists. Suppose the adversary makes a query $E(K, x)$ (either as a normal query or as a part of a super query), and suppose $(K, x, y) \in \mathcal{W}_{\mathrm{tl}} \cup \mathcal{W}_{\mathrm{br}}$ for some $y$. Then, we say that $(K, x, y)$ is wished for, and the wish is granted if the response of the block cipher is $y$. Similar naming is used for inverse queries to $E$. Notice that the adversary may wish for multiple queries at the same time, but this does not invalidate the analysis. Additionally, each wish list element can be wished for only once. In order to find a preimage, the adversary needs at least a wish to be granted. Let $(K, x, y)$ be an element in any of the wish lists, and suppose the adversary makes a query $E(K, x)$ or $E^{-1}(K, y)$. In case of normal queries, the answer is generated from a set of size at least $2^{n-1}$, and the wish is granted with probability at most $\frac{1}{2^{n-1}}$. In case the query is a part of a super query, the answer is generated from a set of size exactly $2^{n-1}$ and the wish is also granted with probability at most $\frac{1}{2^{n-1}}$. Because each element of the wish lists can be wished for only once, the adversary finds a preimage with probability at most

$$
\frac{\left|\mathcal{W}_{\mathrm{tl}}\right|+\left|\mathcal{W}_{\mathrm{tr}}\right|+\left|\mathcal{W}_{\mathrm{bl}}\right|+\left|\mathcal{W}_{\mathrm{br}}\right|}{2^{n-1}}
$$


It remains to bound the sizes of the wish lists after $q$ queries. Configuration $\operatorname{pre}_{\mathrm{tl}}\left(\mathcal{Q}_{q}\right)$ of Fig. 12 has $\leq t_{2}$ solutions for each bl and br (by $\neg$ help $p_{3}\left(\mathcal{Q}_{q}\right)$ ), and consequently $\leq t_{2}$ solutions for $\operatorname{tr}$ (by $\neg$ help $p_{3}\left(\mathcal{Q}_{q}\right)$ ). Thus $\left|\mathcal{W}_{\mathrm{tl}}\right| \leq t_{2}^{3}$, and similarly we obtain $\left|\mathcal{W}_{\mathrm{tr}}\right| \leq t_{2}^{3}$. Configuration $\operatorname{pre}_{\mathrm{bl}}\left(\mathcal{Q}_{q}\right)$ of Fig. 12 has $\leq t_{2}$ solutions for br (by $\neg$ help $\mathrm{p}_{3}\left(\mathcal{Q}_{q}\right)$ ), and consequently $\leq t_{1}$ solutions for $\mathrm{tl}$ (by $\neg$ help $\mathrm{p}_{1}\left(\mathcal{Q}_{q}\right)$ ). For any of these $\leq t_{1} t_{2}$ choices, the query at position $\operatorname{tr}$ is uniquely determined (if it exists at all). Thus $\left|\mathcal{W}_{\mathrm{b}}\right| \leq t_{1} t_{2}$, and similarly $\left|\mathcal{W}_{\text {br }}\right| \leq t_{1} t_{2}$ (using $\neg$ help $p_{2}\left(\mathcal{Q}_{q}\right)$ ). Hence, in this case a preimage is found with probability at most $\frac{4 t_{2}^{3}+4 t_{1} t_{2}}{2^{n}}$.

Case 2. We make the following distinction, and consider the two sub-cases separately.

1. The contributed queries are different for both positions;

2. The contributed queries are the same for both positions.

Case 2.1. In this particular case, the winning query must be a super query. Similar to case 1 , we make use of wish lists, but now for the specific case that a super query contributes two queries to a configuration. As a super query cannot contribute to ( $\mathrm{tl}, \mathrm{tr}$ ), it can only contribute to positions ( $\mathrm{tl}, \mathrm{br}$ ), $(\mathrm{tr}, \mathrm{bl}),(\mathrm{tl}, \mathrm{bl}),(\mathrm{tr}, \mathrm{br})$, or (bl, br). Note that if a super query contributes to positions (tl, br), the left half of the XOR-output of $t \mathrm{l}$ should equal the left half of the key input to br, which is the same as the key input to $\mathrm{tl}$ (similar for super queries contributing to $(\mathrm{tr}, \mathrm{bl})$ ). Note that if a super query contributes to positions ( $\mathrm{tl}, \mathrm{bl})$, the key input to bl equals the key input to $t \mathrm{l}$ which equals the message input to bl (similar for super queries contributing to $(\mathrm{tr}, \mathrm{br})$ ). Also, note that if a super query contributes to positions (bl, br), the XOR-outputs of $\mathrm{tl}$ and tr must be the same. During the attack of the adversary, we maintain five initially empty wish lists $\mathcal{W}_{1}, \ldots, \mathcal{W}_{5}$, corresponding to above cases. If a query is made by the adversary, the wish lists are updated according to the following requirements:

- If the query fits $\operatorname{pre}_{1}(\mathcal{Q})$ of Fig. 13 for any query in the query history, the corresponding tuple $(V \| X, C,(V \| W) \oplus C, B, Z \oplus B)$ is added to $\mathcal{W}_{1}$;

- If the query fits $\operatorname{pre}_{2}(\mathcal{Q})$ of Fig. 13 for any query in the query history, the corresponding tuple $(V \| X, C,(V \| W) \oplus C, A, Y \oplus A)$ is added to $\mathcal{W}_{2}$;

- If the query fits $\operatorname{pre}_{3}(\mathcal{Q})$ of Fig. 13 for any query in the query history, the corresponding tuple $(X\|W, C,(V \| W) \oplus C, X\| W, Y \oplus(X \| W))$ is added to $\mathcal{W}_{3}$;

- If the query fits $\operatorname{pre}_{4}(\mathcal{Q})$ of Fig. 13 for any query in the query history, the corresponding tuple $(X\|W, C,(V \| W) \oplus C, X\| W, Z \oplus(X \| W))$ is added to $\mathcal{W}_{4}$;

- If the query fits $\operatorname{pre}_{5}(\mathcal{Q})$ of Fig. 13 for any query in the query history, the corresponding tuple $(W \| X, A, Y \oplus A, B, Z \oplus B)$ is added to $\mathcal{W}_{5}$.

Of these tuples, the first element identifies the key for which the super query is made, the second and third element define the input and output of the cipher in the top row (either left or right), and the fourth and fifth element define the input and output of the cipher in the bottom row (either right or left). For $\mathcal{W}_{5}$, the second and third element correspond to bl and the fourth and fifth element to br. A query trivially does not add itself to the wish list (as these cases would be covered by Case 3 ). Suppose the adversary makes a super query to $E$ for key $K$, and suppose $\left(K, x_{\mathrm{tl}}, y_{\mathrm{t}}, x_{\mathrm{br}}, y_{\mathrm{br}}\right) \in \mathcal{W}_{1}$ for some $x_{\mathrm{tl}}, y_{\mathrm{tl}}, x_{\mathrm{br}}, y_{\mathrm{br}}$. This wish is then granted if the response satisfies $y_{\mathrm{tl}}=E\left(K, x_{\mathrm{tl}}\right)$ and $y_{\mathrm{br}}=E\left(K, x_{\mathrm{br}}\right)$. In order to find a preimage, the adversary needs at least a wish to be granted. As the answers are generated from a set of size exactly $2^{n-1}$, a wish is granted with probability at most $\frac{1}{2^{n-1}\left(2^{n-1}-1\right)}$. Because each element of the wish lists can be wished for only once, the adversary finds a preimage with probability at most

$$
\frac{\left|\mathcal{W}_{1}\right|+\left|\mathcal{W}_{2}\right|+\left|\mathcal{W}_{3}\right|+\left|\mathcal{W}_{4}\right|+\left|\mathcal{W}_{5}\right|}{2^{n-1}\left(2^{n-1}-1\right)}
$$

It remains to bound the sizes of the wish lists after $q$ queries. Configuration $\operatorname{pre}_{1}\left(\mathcal{Q}_{q}\right)$ has $\leq t_{2}$ solutions for bl (by $\neg$ help $\mathrm{p}_{3}\left(\mathcal{Q}_{q}\right)$ ), and at most $\leq t_{1}$ solutions for $\operatorname{tr}\left(\right.$ by $\neg$ help $p_{1}\left(\mathcal{Q}_{q}\right)$ ). Thus, $\left|\mathcal{W}_{1}\right| \leq t_{1} t_{2}$ and similarly $\left|\mathcal{W}_{2}\right| \leq t_{1} t_{2}$. Configuration $\operatorname{pre}_{3}\left(\mathcal{Q}_{q}\right)$ has $\leq t_{2}$ solutions for br (by $\neg$ help $p_{3}\left(\mathcal{Q}_{q}\right)$ ), and at most $\leq t_{1}$ solutions for $\operatorname{tr}\left(\right.$ by $\neg$ help $p_{2}\left(\mathcal{Q}_{q}\right)$ ). Additionally, there are $2^{n / 2}$ possibilities for $W$. Thus $\left|\mathcal{W}_{3}\right| \leq t_{1} t_{2} 2^{n / 2}$ and similarly $\left|\mathcal{W}_{4}\right| \leq t_{1} t_{2} 2^{n / 2}$. Configuration $\operatorname{pre}_{5}\left(\mathcal{Q}_{q}\right)$ has $2^{n / 2}$ choices for $W$, for any of these choices it has $\leq t_{1}$ 

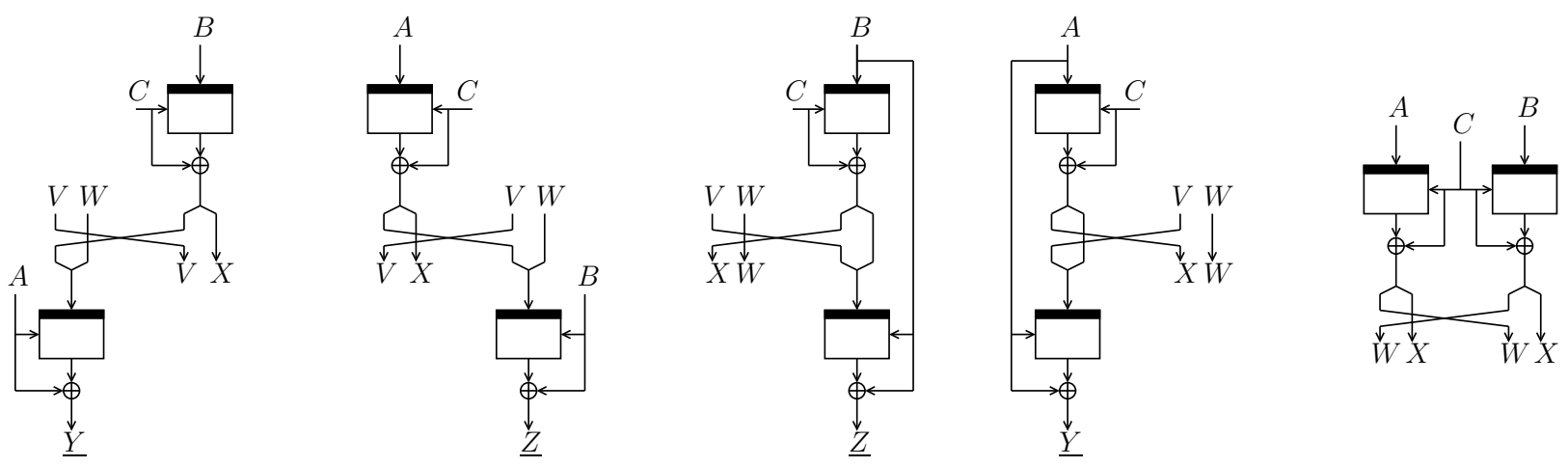

Fig. 13. From left to right: configurations $\operatorname{pre}_{1}(\mathcal{Q}), \ldots, \operatorname{pre}_{5}(\mathcal{Q})$.

solutions for $\operatorname{tr}$ (by $\neg$ help $p_{1}\left(\mathcal{Q}_{q}\right)$ ), and consequently $\leq t_{2}$ solutions for $\mathrm{tl}$ (by $\neg \operatorname{hel}_{3}\left(\mathcal{Q}_{q}\right)$ ). Thus also $\left|\mathcal{W}_{5}\right| \leq t_{1} t_{2} 2^{n / 2}$. Hence, in this case a preimage is found with probability at most $\frac{16 t_{1} t_{2}+24 t_{1} t_{2} 2^{n / 2}}{2^{2 n}}$.

Case 2.2. In this case, the winning query may be a normal query or a super query. The winning query can only contribute to positions $(\mathrm{tl}=\mathrm{br}),(\mathrm{tr}=\mathrm{bl}),(\mathrm{tl}=\mathrm{bl})$, or $(\mathrm{tr}=\mathrm{br})$.

We first consider the winning query to contribute to $\mathrm{tl}=\mathrm{br}$. Suppose the adversary makes a query $y \leftarrow E(K, x)$ (either as a normal query or as a part of a super query). As it occurs at position br we require $x \oplus y=Z$ (because of this, the analysis for inverse queries is equivalent). Additionally, the query at position tr should have key input as well as message input equal to $x$. This particularly means that the key input $K$ to $\mathrm{tl}=$ br must satisfy $K=Z^{l} \|(E(x, x) \oplus x)^{r}$. By construction of the queries at positions bl, br, the adversary can only succeed if it ever finds an $x \in \mathbb{Z}_{2}^{n}$ that satisfies

$$
\begin{aligned}
& E\left((E(x, x) \oplus x)^{l}\left\|Z^{r}, Z^{l}\right\|(E(x, x) \oplus x)^{r}\right) \oplus Z^{l} \|(E(x, x) \oplus x)^{r}=Y, \\
& E\left(Z^{l} \|(E(x, x) \oplus x)^{r}, x\right) \oplus x=Z .
\end{aligned}
$$

As $Y$ and $Z$ are fixed, the adversary finds such $x$ with probability at most $\frac{2^{n}}{2^{n-1} 2^{n-1}}=\frac{4}{2^{n}}$. The same probability bound is obtained for winning queries to appear at (tr, bl).

Consider a query contributing to $\mathrm{t} \mathrm{l}=\mathrm{bl}$. By construction, this query must be of the form $E(K, K)=y$ where $K \oplus y=Y$ and $K^{r}=Y^{r}$. As $Y$ is fixed, the adversary finds such query with probability at most $\frac{2^{n / 2}}{2^{n-1}}=\frac{2 \cdot 2^{n / 2}}{2^{n}}$ (either in case of forward or inverse query). The same probability bound is obtained for winning queries to appear at (tr, br).

Consequently, a preimage is found in this case with probability at most $\frac{8+4 \cdot 2^{n / 2}}{2^{n}}$.

Case 3. Recall that a query can never contribute to ( $\mathrm{tl}, \mathrm{tr}$ ) at the same time. Therefore, we only need to consider queries contributing at positions ( $\mathrm{tl}, \mathrm{bl}, \mathrm{br}$ ) or ( $\mathrm{tr}, \mathrm{bl}, \mathrm{br}$ ). We make the following distinction, and consider the two sub-cases separately.

1. The contributed queries are different for all positions;

2. The contributed queries are the same at two positions.

Note that as the queries at (bl, br) cannot be the same, there is no need to consider the case all three queries are the same.

Case 3.1. As before, we consider two wish lists $\mathcal{W}_{\mathrm{tl}}, \mathcal{W}_{\mathrm{tr}}$, corresponding to position to which the winning query does not contribute. Note that if a super query contributes to positions (tr, bl, br), the $\mathrm{XOR}$-outputs of $\mathrm{tl}$ and $\mathrm{tr}$ must be the same, and equal to the key input to tr. In order words, any query to $\mathrm{tl}$ fixes exactly one wish list tuple in $\mathcal{W}_{\mathrm{tl}}$. In more detail, if a query $E(K, x)=y$ is made the wish lists are updated as follows:

- The tuple $(x \oplus y, x, y, K, Y \oplus K, x \oplus y, Z \oplus x \oplus y)$ is added to $\mathcal{W}_{\mathrm{tl}}$;

- The tuple $(x \oplus y, x, y, x \oplus y, Y \oplus x \oplus y, K, Z \oplus K)$ is added to $\mathcal{W}_{\text {tr. }}$.

Of these tuples, the first element identifies the key, the second and third element define the input and output of the cipher in the top row (either left or right), the fourth and fifth element define the input 
and output of the cipher at bl and the sixth and seventh element the input and output of the cipher at br. Because each element of the wish lists can be wished for only once, the adversary finds a preimage with probability at most

$$
\frac{\left|\mathcal{W}_{\mathrm{t} \mid}\right|+\left|\mathcal{W}_{\mathrm{tr}}\right|}{2^{n-1}\left(2^{n-1}-1\right)\left(2^{n-1}-2\right)}
$$

Clearly, $\left|\mathcal{W}_{\mathrm{t}}\right|,\left|\mathcal{W}_{\mathrm{tr}}\right| \leq q$. Hence, in this case a preimage is found with probability at most $\frac{64 q}{2^{3 n}}$.

Case 3.2. Note that the same query can only occur at positions $(\mathrm{tl}=\mathrm{br}),(\mathrm{tr}=\mathrm{bl}),(\mathrm{tl}=\mathrm{bl})$, or $(\mathrm{tr}=\mathrm{br})$. The analysis of case 2.2 carries over directly, but for the latter two scenarios we can do better. Consider a query contributing to $\mathrm{tl}=\mathrm{bl}$. Note that, as the super query also contributes to position $\mathrm{br}$, the key inputs to $\mathrm{tl}$, bl, br are the same. By construction, the query at $\mathrm{tl}=\mathrm{bl}$ must be of the form $E(K, K)=y$ where $K \oplus y=Y$ and $K=Y$. As $Y$ is fixed, the adversary finds such query with probability at most $\frac{1}{2^{n-1}}=\frac{2}{2^{n}}$ (either in case of forward or inverse query). The same probability bound is obtained for winning queries to appear at $(\operatorname{tr}=\mathrm{br})$. Consequently, a preimage is found in this case with probability at most $\frac{12}{2^{n}}$.

The claim is obtained by summing the bounds obtained for the three cases.

\section{B.2 Proof of Lem. 10}

It suffices to consider the events $\operatorname{Pr}\left(\operatorname{help}_{k}\left(\mathcal{Q}_{q}\right)\right)(k=1,2,3)$ separately.

$\operatorname{help}_{k}\left(\mathcal{Q}_{q}\right)$ for $k \in\{1,2\}$. The cases are equivalent by symmetry, and we consider help ${ }_{1}\left(\mathcal{Q}_{q}\right)$ only. Let $z \in \mathbb{Z}_{2}^{n / 2}$. Denote by $\mathcal{Q}_{q}^{(n)}$ the restriction of $\mathcal{Q}_{q}$ to normal queries, and by $\mathcal{Q}_{q}^{(s)}$ the restriction of $\mathcal{Q}_{q}$ to queries that belong to super queries. In order for $\mathcal{Q}_{q}$ to have more than $t_{1}$ solutions to $\left(x_{i} \oplus y_{i}\right)^{l}=z$, at least one of the following criteria needs to hold:

1. $\mathcal{Q}_{q}^{(n)}$ has more than $t_{1} / 2$ solutions;

2. $\mathcal{Q}_{q}^{(s)}$ has more than $t_{1} / 2$ solutions.

We consider these two scenarios separately. In case of normal queries, each query $\left(K_{i}, x_{i}, y_{i}\right)$ is answered with a value generated at random from a set of size at least $2^{n-1}$, and hence it satisfies $\left(x_{i} \oplus y_{i}\right)^{l}=z$ with probability at most $\frac{2^{n / 2}}{2^{n-1}}=\frac{2}{2^{n / 2}}$. More than $t_{1} / 2$ queries result in a solution with probability at $\operatorname{most}\left(\begin{array}{c}q \\ t_{1} / 2\end{array}\right)\left(\frac{2}{2^{n / 2}}\right)^{t_{1} / 2} \leq\left(\frac{4 e q}{t_{1} 2^{n / 2}}\right)^{t_{1} / 2}$.

The analysis for super queries is more elaborate. In order for $\mathcal{Q}_{q}^{(s)}$ to have more than $t_{1} / 2$ solutions, as at most $q / 2^{n-1}$ super queries occur, at least one of the super queries needs to provide more than $t_{1}^{\prime}:=\frac{t_{1}}{2 q / 2^{n-1}}=\frac{t_{1} 2^{n}}{4 q}$ solutions. Consider any super query, consisting of $2^{n-1}$ queries. It provides more than $t_{1}^{\prime}$ solutions with probability at most

$$
\left(\begin{array}{c}
2^{n-1} \\
t_{1}^{\prime}
\end{array}\right) \prod_{j=0}^{t_{1}^{\prime}-1} \frac{2^{n / 2}}{2^{n-1}-j} \leq\left(\begin{array}{c}
2^{n-1} \\
t_{1}^{\prime}
\end{array}\right)\left(\frac{2^{n / 2}}{2^{n-1}-t_{1}^{\prime}}\right)^{t_{1}^{\prime}} \leq\left(\frac{e 2^{n-1} 2^{n / 2}}{t_{1}^{\prime}\left(2^{n-1}-t_{1}^{\prime}\right)}\right)^{t_{1}^{\prime}} .
$$

Provided $t_{1} \leq q$, we have $t_{1}^{\prime}=\frac{t_{1} 2^{n}}{4 q} \leq 2^{n-2}$, and thus $\frac{1}{2^{n-1}-t_{1}^{\prime}} \leq \frac{1}{2^{n-2}}$. Consequently, this super query adds more than $\frac{t_{1} 2^{n}}{4 q}$ solutions with probability at most $\left(\frac{8 e q}{t_{1} 2^{n / 2}}\right)^{\frac{t_{1} 2^{n}}{4 q}}$. In order to cover any super query, we need to multiply this probability with $q / 2^{n-1}$.

Considering any possibly choice for $z$, we obtain for $k=1,2$ :

$$
\operatorname{Pr}\left(\operatorname{help}_{k}\left(\mathcal{Q}_{q}\right)\right) \leq 2^{n / 2}\left(\frac{4 e q}{t_{1} 2^{n / 2}}\right)^{t_{1} / 2}+2^{n / 2} \cdot \frac{q}{2^{n-1}}\left(\frac{8 e q}{t_{1} 2^{n / 2}}\right)^{\frac{t_{1} 2^{n}}{4 q}} .
$$

help ${ }_{3}\left(\mathcal{Q}_{q}\right)$. A similar analysis as for help ${ }_{1}\left(\mathcal{Q}_{q}\right)$ results in the following bound:

$$
\operatorname{Pr}\left(\operatorname{help}_{3}\left(\mathcal{Q}_{q}\right)\right) \leq 2^{n}\left(\frac{4 e q}{t_{2} 2^{n}}\right)^{t_{2} / 2}+2^{n} \cdot \frac{q}{2^{n-1}}\left(\frac{8 e q}{t_{2} 2^{n}}\right)^{\frac{t_{2} 2^{n}}{4 q}}
$$

The claim is obtained by adding (18) (twice) and (19). 